\title{
NON-AUTONOMOUS \\ CONFORMAL ITERATED FUNCTION SYSTEMS AND MORAN-SET CONSTRUCTIONS
}

\author{
LASSE REMPE-GILLEN AND MARIUSZ URBAŃSKI
}

\begin{abstract}
We study non-autonomous conformal iterated function systems, with finite or countable infinite alphabet alike. These differ from the usual (autonomous) iterated function systems in that the contractions applied at each step in time are allowed to vary. (In the case where all maps are affine similarities, the resulting system is also called a "Moran set construction".)

We shall show that, given a suitable restriction on the growth of the number of contractions used at each step, the Hausdorff dimension of the limit set of such a system is determined by an equation known as Bowen's formula. We also give examples that show the optimality of our results.

In addition, we prove Bowen's formula for a class of infinite alphabet-systems and deal with Hausdorff and packing measures for finite systems, as well as continuity of topological pressure and Hausdorff dimension for both finite and infinite systems. In particular we strengthen the existing continuity results for infinite autonomous systems.

As a simple application of our results, we show that, for a transcendental meromorphic function $f$, the Hausdorff dimension of the set of transitive points (i.e., those points whose orbits are dense in the Julia set) is bounded from below by the hyperbolic dimension of $f$ (in the sense of Shishikura).
\end{abstract}

\section{INTRODUCTION}

1.1. Background. In the classical theory of iterated function systems, one is given a finite set $\Phi=\left\{\varphi_{i}\right\}_{i \in I}$ of uniformly contracting affine similarities $\varphi_{i}: \mathbb{R}^{d} \rightarrow \mathbb{R}^{d}$ and studies the limit set $J=J(\Phi)$ of the system $\Phi$, defined as the set of possible limit points of sequences $\varphi_{\omega_{1}}\left(\varphi_{\omega_{2}}\left(\ldots\left(\varphi_{\omega_{n}}(x)\right) \ldots\right)\right)$, with $x \in \mathbb{R}^{d}$ and $\omega_{j} \in I$ for all $j$. Famous examples of such "self-similar" sets include the Middle-third Cantor set, the Sierpiński triangle, the van Koch curve, and many others.

The theory of systems where the contractions $\varphi_{i}$ are not necessarily affine similarities but merely conformal, and where the alphabet $I$ is not necessarily finite but only required to be countable, is also well developed; see e.g. [9] (which laid the foundation for the theory of infinite systems), [10, and [4].

From the point of view of dynamical systems, the above represent an autonomous setting: the rule applied to the system is independent of the time $n$. The analogy to ordinary dynamical systems is particularly transparent if the ranges of all contractions $\varphi_{i}, i \in I$, are mutually disjoint. Then one can consider the dynamics on $J(\Phi)$ given by the inverses of the functions $\varphi_{i}: J \rightarrow \varphi_{i}(J)$. This gives an expanding non-invertible

2010 Mathematics Subject Classification. 28A80 (Primary); 37C45, 37F10 (Secondary).

The first author was supported by EPSRC Fellowship EP/E052851/1. The second author was supported in part by NSF Grant DMS 1001874. 
dynamical system on the limit set. Conversely, we may often pass from a non-invertible dynamical system $f$ to an iterated function system whose maps $\varphi_{i}$ are inverse branches of iterates of $f$.

The first and often most important question regarding an autonomous conformal iterated function system $\Phi$ is how to determine the Hausdorff dimension $\operatorname{HD}(J(\Phi))$ of the limit set $J(\Phi)$. In the classical setting described above, it is well-known that this dimension is given by the solution $t$ to the equation $\sum_{i \in I}\left\|D \varphi_{i}\right\|^{t}=1$. For a general autonomous conformal iterated function system, the number $t$ generalizes to the Bowen dimension $B(\Phi)$. Informally speaking, $B(\Phi)$ is the number that is obtained by modifying the usual definition of Hausdorff dimension to allow only the natural coverings of $J(\Phi)$ corresponding to the $n$-th levels in the iterative construction. Then Bowen's formula states that $\operatorname{HD}(J(\Phi))=B(\Phi)$. The pioneer work here belongs to Moran [11] and Bowen [1], while Bowen's formula was proved in [10] for general infinite autonomous conformal iterated autonomous iterated function systems (and even more generally "graph directed Markov systems").

Non-autonomous systems. It is natural, and frequently necessary in applications, to consider the non-autonomous version of the above setting, where the system $\Phi$ is allowed to vary with time $n$. More precisely, the system $\Phi$ consists of a sequence $\left(\Phi^{(n)}\right)_{n \in \mathbb{N}}$ of collections of conformal contractions, where $\Phi^{(n)}=\left\{\varphi_{i}^{(n)}\right\}_{i \in I^{(n)}}$ may vary with $n$. The limit set $J(\Phi)$ now consists of the possible limits of sequences $\left.\varphi_{\omega 1}^{(1)}\left(\varphi_{\omega_{2}}^{(2)}\left(\cdots \circ \varphi_{\omega_{n}}^{(n)}(x)\right) \ldots\right)\right)$, where $\omega_{j} \in I^{(j)}$ for all $j$.

In the case where all $\varphi_{i}^{(n)}$ are affine similarities, this is also referred to as a Moran set construction 1 This entire paper is devoted to study conformal non-autonomous systems. However, we also touch on autonomous systems: On the one hand, they occur as auxiliary objects in our non-autonomous constructions, on the other hand, we also prove new meaningful theorems about autonomous systems.

Our central objective is to establish versions of Bowen's formula that are as general as possible. But we will also touch on other aspects, such as the continuity of Hausdorff dimension and the relationship of nonautonomous systems to random iterated function systems as introduced in [14].

Bowen's Formula. The definition of the Bowen dimension $B(\Phi)$ generalizes naturally to non-autonomous systems (see Definition 2.7). However, in this setting Bowen's formula $\operatorname{HD}(J(\Phi))=B(\Phi)$ no longer holds in general. (This follows from our theorems below, but has been known for a long time; compare e.g. [19.) Our key observation for finite systems (i.e., those for which all index sets $I^{(n)}$ are finite) is that Bowen's formula does hold when we restrict the growth of the size $\# I^{(n)}$ of the $n$-th level index sets. Throughout the article, the dimension of the ambient space is denoted by $d$.

\footnotetext{
${ }^{1}$ Moran set constructions are somewhat more general than our non-autonomous systems, in that the placement of pieces at level $n+1$ is allowed to occur independently for each level $n$ piece. All our results (and proofs) will remain true in this somewhat more general set-up. However, we feel that our setting is natural from the point of view of dynamics, and prefer not to increase the notational complexity further.
} 
1.1. Theorem (Bowen's formula for systems of sub-exponential growth). Suppose that $\Phi$ is a non-autonomous conformal iterated function system of sub-exponential growth:

$$
\lim _{n \rightarrow \infty} \frac{1}{n} \log \# I^{(n)}=0 .
$$

Then Bowen's Formula holds for $\Phi$, i.e. $\operatorname{HD}(J(\Phi))=B(\Phi)$.

On the other hand, suppose that $0<t_{1}<t_{2}<d$ and let $\varepsilon>0$. Then there exists a non-autonomous conformal iterated function system $\Phi$ such that

$$
\limsup _{n \rightarrow \infty} \frac{1}{n} \log \# I^{(n)} \leq \varepsilon,
$$

$\mathrm{HD}(J(\Phi))=t_{1}$ and $B(\Phi)=t_{2}$.

The counterexamples we construct to prove the second part of the theorem are very irregular: there are many stages consisting of pieces of definite size, but at some stages the number of pieces is large. Also, the examples cannot be extended to the case $t_{1}=0$ or $t_{2}=d$. The following theorem shows that both of these restrictions are necessary.

1.2. Theorem (Bowen's formula for some systems of exponential growth). Suppose that $\Phi$ is a non-autonomous conformal iterated function system of at most exponential growth:

$$
\limsup _{n \rightarrow \infty} \frac{1}{n} \log \# I^{(n)}<\infty .
$$

Suppose additionally that one of the following is true:

(a) $\left\|D \varphi_{i}^{(n)}\right\| \rightarrow 0$ uniformly in $i$ as $n \rightarrow \infty$,

(b) $\operatorname{HD}(J(\Phi))=0$, or

(c) $B(\Phi)=d$.

Then Bowen's Formula holds for $\Phi$, i.e. $\operatorname{HD}(J(\Phi))=B(\Phi)$.

On the other hand, let $\left(\alpha_{n}\right)_{n \in \mathbb{N}}$ be an arbitrary sequence of positive integers such that $\lim _{n \rightarrow \infty} \log \alpha_{n} / n=\infty$. Then there exists a non-autonomous conformal iterated function system $\Phi$ such that $\# I^{(n)} \leq \alpha_{n}$ for all $n$, and such that (回) to (c) hold for $\Phi$.

The idea of proof for both of the preceding theorems is as follows. We prove a general lower bound on $\operatorname{HD}(\Phi)$ for a finite non-autonomous conformal iterated function system, and use this bound to establish the above results assuming additional "balancing" assumptions (meaning that we restrict how much the pieces at a given level may differ in size). We are then able to remove those assumptions by studying suitable subsystems (this is an idea that stems from the study of infinite iterated function systems; the novelty of our approach lies in its application to finite cases).

A special case of Theorem 1.2 concerns systems of sufficiently regular exponential growth:

1.3. Proposition (Regular exponential growth). Suppose that $\Phi$ a system such that both limits

$$
a:=\lim _{n \rightarrow \infty} \frac{1}{n} \log \# I^{(n)}
$$

and

$$
b:=\lim _{n \rightarrow \infty, j \in I^{(n)}} \frac{1}{n} \log \left(1 /\left\|D \varphi_{j}^{(n)}\right\|\right)
$$


exist and are finite and positive. Then $B(\Phi)=a / b$, and hence $\operatorname{HD}(J(\Phi))=B(\Phi)=a / b$.

As an application of our results, let $\lambda>1$ and consider the set of real numbers $x \in[0,1]$ whose continued fraction expansion $x=\left[a_{0}, a_{1}, a_{2}, \ldots\right]$ satisfies $\lambda^{n}<a_{n}<\lambda^{n+1}$ for sufficiently large $n$. It follows from a result of Jordan and Rams [7] that this set has Hausdorff dimension 1/2. We can also see this directly from the above Proposition; indeed, here we have

$$
a=\lim _{n \rightarrow \infty} \frac{1}{n} \log \left(\lambda^{n+1}-\lambda^{n}\right)=\log \lambda
$$

and

$$
b=\lim _{n \rightarrow \infty} \frac{1}{n} \log \lambda^{2(n+1)}=2 \log \lambda .
$$

Having established Bowen's formula for finite systems we also prove a version for a large class sufficiently regular infinite ones. This is provided by Corollary 8.3 in Section 8 (Systems with Countably Infinite Alphabet). We also briefly comment on the relation of our results to the study of random iterated function systems, as well as the continuity of pressure and Hausdorff dimension (following the line of research initiated in [14] and continued in [15] in the context of autonomous systems). The latter is done under considerably weaker hypotheses than in [14] giving stronger results even for autonomous systems.

In an appendix, we provide a simple and useful application of our methods to complex dynamics:

1.4. Theorem (Transitive points of meromorphic functions). Let $f: \mathbb{C} \rightarrow \hat{\mathbb{C}}$ be a nonlinear, non-constant meromorphic function, and let $J_{\text {dense }}$ denote the set of transitive points. That is, $J_{\text {dense }}$ consists of those points in the Julia set $J(f)$ whose orbit is dense in $J(f)$.

Then $\mathrm{HD}\left(J_{\text {dense }}(f)\right) \geq \operatorname{HD}_{\text {hyp }}(f)$, where $\operatorname{HD}_{\text {hyp }}(f)$ denotes the hyperbolic dimension of $f$ in the sense of Shishikura.

(We also prove a version of this result for autonomous infinite iterated function systems.)

Previous and related results. Non-autonomous systems and Moran-set constructions have been studied by a variety of authors previously, and we cannot give a survey here; instead we shall mention a small number of papers that are particularly relevant to our study.

The article [19] from 2001 presents a survey of results known at the time. Zhi-Ying Wen has kindly pointed out that, in the case where $d=1$ and all maps $\varphi_{i}^{(n)}$ are affine similarities, a slightly stronger result than the positive part of Theorem 1.1 appears in [2, Theorem 2]. However, the proof appears to use the one-dimensionality of the phase space in an essential way.

We would also like to mention recent results independently obtained by Holland and Zhang [5]. Similarly to us, they consider a type of Moran set construction in $\mathbb{R}^{d}$ and establish Bowen's formula (as well as formulae for the packing dimension) in certain cases. However, while there is some overlap between the results, [5] takes a different route from the present article. In particular, the results there assume some control over 
the minimal contraction factors, and in particular do not imply our theorems stated in the introduction.

Structure of the paper. Section 2 contains the definitions that are fundamental for our study: non-autonomous systems, words, limit sets, lower pressure and Bowen dimension. In Section 3 we establish a general lower bound on the Hausdorff dimension of the limit set of a finite non-autonomous conformal iterated functions system, which is then used in Section 4 to prove preliminary versions of Theorems 1.1 and 1.2 above. The proof of both theorems is completed in Sections 5 and 6 by considering suitable subsystems for the positive direction, and by discussing the construction of counterexamples to Bowen's formula that establish the optimality of our results. Completing our treatment of finite systems, we briefly discuss Hausdorff and packing measures of limit sets for a class of uniformly finite systems in Section 7.

In Section 8 , we use the approximation by subsystems developed in Section 5 to prove Bowen's formula for some large classes of infinite systems. Random iterated function systems are discussed in Section 9, and continuity of pressure and Hausdorff dimension is treated in Section 10.

We discuss the above-mentioned application to transitive points in complex dynamics and in infinite iterated function systems in an Appendix.

Acknowledgements. We would like to thank Mark Holland, Thomas Jordan and ZhiYing Wen for interesting discussions about this work. We are also grateful to the referee for their careful reading of the paper and a multitude of corrections and helpful suggestions.

\section{Definitions And First Estimates}

In this section, we define the class of non-autonomous conformal iterated function systems, which we shall abbreviate as NCIFS, in a Euclidean space $\mathbb{R}^{d}$. Our results will be stated and proved in this setting. We essentially follow the notation for infinite iterated function systems in [10]. We also define the upper and lower pressure functions for such systems, and derive some of their basic general properties. To conclude the section, we establish an elementary upper bound for the Hausdorff dimension of the limit set of any non-autonomous conformal iterated function system. This is the easier part of Bowen's formula.

Throughout the article, we fix $d \in \mathbb{N}$ and a compact set $X \subset \mathbb{R}^{d}$ with $\overline{\operatorname{int}(X)}=X$, with the additional geometric assumption that $\partial X$ is smooth or that $X$ is convex. (More generally, we can allow sets that satisfy the more technical condition (2.7) from [9].)

Given a conformal map $\varphi: X \rightarrow X$ we denote by $\varphi_{i}^{\prime}(x)$ or by $D \varphi_{i}(x)$ the derivative of $\varphi$ evaluated at $x$, i.e. $\varphi_{i}^{\prime}(x): \mathbb{R}^{d} \rightarrow \mathbb{R}^{d}$ is a similarity linear map, and we denote by $\left|\varphi_{i}^{\prime}(x)\right|$ (or by $\left|D \varphi_{i}(x)\right|$ ) its scaling factor. We also put

$$
\|D \varphi\|=\left\|\varphi^{\prime}\right\|=\sup \left\{\left|\varphi^{\prime}(x)\right|: x \in X\right\} .
$$

\subsection{Definition.}

A non-autonomous conformal iterated function system (NCIFS) $\Phi$ on the set $X$ is given by a sequence $\Phi^{(1)}, \Phi^{(2)}, \Phi^{(3)}, \ldots$, where each $\Phi^{(j)}$ is a set of functions $\left(\varphi_{i}^{(j)}: X \rightarrow X\right)_{i \in I^{(j)}}$ and each $I^{(j)}$ is a (finite or countably infinite) index set, if the following hold. 
(a) Open set condition: We have

$$
\varphi_{a}^{(j)}(\operatorname{int}(X)) \cap \varphi_{b}^{(j)}(\operatorname{int}(X))=\emptyset
$$

for all $j \in \mathbb{N}$ and all distinct indices $a, b \in I^{(j)}$.

(b) Conformality: There exists an open connected set $V \supset X$ (independent of $i$ and $j$ ) such that each $\varphi_{i}^{(j)}$ extends to a $C^{1}$ conformal diffeomorphism of $V$ into $V$.

(c) Bounded distortion: There exists a constant $K \geq 1$ such that, for any $k \leq l$ and any $\omega_{k}, \omega_{k+1}, \ldots, \omega_{l}$ with $\omega_{j} \in I^{(j)}$, the map $\varphi:=\varphi_{\omega_{k}} \circ \cdots \circ \varphi_{\omega_{l}}$ satisfies

$$
\|D \varphi(x)\| \leq K\|D \varphi(y)\|
$$

for all $x, y \in V$.

(d) Uniform contraction: There is a constant $\eta<1$ such that

$$
\|D \varphi(x)\| \leq \eta^{m}
$$

for all sufficiently large $m$, all $x \in X$ and all $\varphi=\varphi_{\omega_{j}} \circ \cdots \circ \varphi_{\omega_{j+m}}$, where $j \geq 1$ and $\omega_{k} \in I^{(k)}$. In particular, this holds if

$$
\left\|D \varphi_{i}^{(j)}(x)\right\| \leq \eta
$$

for all $j \geq 1$ and all $x \in X$, which we assume in the sequel for the ease of exposition.

The system $\Phi$ is called autonomous if $I^{(n)}$ and $\Phi^{(n)}$ are independent of $n$.

Remark. We remark that condition (c) (bounded distortion) is automatically satisfied when $d \geq 2$. Indeed, for $d \geq 3$ this condition can be deduced from the celebrated Liouville Theorem asserting that each conformal map is a composition of an inversion with respect to some sphere (perhaps of infinite radius), a Euclidean linear similarity and a translation. In the case when $d=2$ any conformal map is either holomorphic or anti-holomorphic and condition (c) can be easily deduced from the celebrated Koebe Distortion Theorem.

Hence condition (c) only needs to be verified in the case $d=1$. Of course bounded distortion is always satisfied (with $K=1$ ) if the system consists of similarities.

For the definitions and discussions that follow, we fix some nonautonomous conformal IFS $\Phi$. The combinatorial language we introduce will be used throughout the article.

\subsection{Definition (Words).}

We define symbolic spaces, for $0<m \leq n<\infty$ by

$$
I^{n}:=\prod_{j=1}^{n} I^{(j)}, \quad I^{\infty}:=\prod_{j=1}^{\infty} I^{(j)}, \quad I^{m, n}:=\prod_{j=m}^{n} I^{(j)} \quad \text { and } \quad I^{m, \infty}:=\prod_{j=m}^{\infty} I^{(m)} .
$$

Elements of $I^{n}$ (with $n \leq \infty$ ) are called (initial) words, while those of $I^{m, n}$ with $m>1$ are called non-initial words. We assume all words are initial, unless explicitly stated otherwise.

The length of a word $\omega \in I^{m, n}$ is $|\omega|:=n-m$; $\omega$ is called finite or infinite according to whether its length is finite or infinite. Finite words are sometimes also referred to as blocks. 
If $\omega=\omega_{m} \omega_{m+1} \ldots \omega_{n} \in I^{m, n}$ is a finite word, we define the associated conformal map by

$$
\varphi_{\omega}^{m, n}:=\varphi_{\omega_{m}}^{(m)} \circ \cdots \circ \varphi_{\omega_{n}}^{(n)} .
$$

In the case of an initial word, where $m=1$, we also abbreviate $\varphi_{\omega}:=\varphi_{\omega}^{n}:=\varphi_{\omega}^{1, n}$.

Remark. We note the similarity of notation between the individual index sets $I^{(n)}$ and the $n$-fold product $I^{n}$. No confusion should arise, as it will always be clear from context whether we mean symbols or words.

We can now define the central object of study: the limit set of a nonautonomous IFS.

2.3. Definition (Limit set).

For all $n \in \mathbb{N}$ and $\omega \in I^{n}$, we define

$$
X_{\omega}:=\varphi_{\omega}^{n}(X) \quad \text { and } \quad X_{n}:=\bigcup_{\omega \in I^{n}} X_{\omega} .
$$

The limit set (or attractor) of $\Phi$ is defined as

$$
J:=J(\Phi):=\bigcap_{n=1}^{\infty} X_{n} .
$$

Remark. In the case where all sets $I^{(j)}, j \geq 1$, are finite, the limit set $J(\Phi)$ is compact, and hence closed, as an intersection of compact sets. If some of the sets $I^{(j)}$ are infinite, the limit set $J(\Phi)$ is not closed in general, and its closure can be much bigger than $J(\Phi)$ - both topologically and with respect to Hausdorff dimension. Indeed, it is not difficult to construct infinite autonomous systems such that $J(\Phi)$ is dense in $X$ but has Hausdorff dimension equal to zero.

There is another useful description of the limit set. If $\omega \in I^{m, n}$ (where $n$ is either finite or infinite) and $s \leq|\omega|$, then by $\left.\omega\right|_{s}$ we mean the word

$$
\omega_{m} \omega_{m+1} \ldots \omega_{m+s-1} \in I^{m, m+s-1},
$$

i.e. the initial subword of $\omega$ of length $s$. If $\omega \in I^{\infty}$, then $\left(\varphi_{\left.\omega\right|_{n}}(X)\right)_{n=1}^{\infty}$ is a descending sequence of compact sets whose diameters converge to zero exponentially fast. Indeed, the uniform contraction assumption means that

$$
\left\|\varphi_{\omega}^{\prime}\right\| \leq \eta^{n}
$$

and hence

$$
\operatorname{diam}\left(\varphi_{\omega}(X)\right) \leq \operatorname{const} \eta^{n}
$$

for sufficiently large $n$ and all $\omega \in I^{n}$.

2.4. Definition and Lemma (The projection map). Define

$$
\pi_{\Phi}: I^{\infty} \rightarrow J(\Phi) ; \quad\left\{\pi_{\Phi}(\omega)\right\}:=\bigcap_{n=1}^{\infty} \varphi_{\left.\omega\right|_{n}}(X) .
$$

Then

$$
J(\Phi)=\pi_{\Phi}\left(I^{\infty}\right)
$$


Proof. As noted above, the diameter of $\varphi_{\left.\omega\right|_{n}}(X)$ tends to zero as $n \rightarrow \infty$, hence $\pi_{\Phi}$ is indeed a well-defined function, whose values belong to $J(\Phi)$ by definition.

To prove the converse, let $x \in J(\Phi)$. We use the fact that, for every $n \in \mathbb{N}$, the set

$$
\left\{\omega \in I^{n}: x \in X_{\omega}\right\}
$$

is finite. This is trivial when each index set is finite, but otherwise it uses the open set condition together with the fact that - due to the geometric assumptions on the boundary of $X$ - each set $X_{\omega}$ with $x \in X_{\omega}$ contains a cone of definite opening angle based at $x$ (see [9, Formula (2.10)]).

Now form a directed graph on the set of finite initial words $\omega$ with $x \in X_{\omega}$, by drawing a directed edge from $\omega^{1}$ to $\omega^{2}$ if and only if $\omega^{1}$ is obtained from $\omega^{2}$ by deleting the last symbol. By assumption, this graph contains arbitrarily long directed paths that start at the empty word, and by the above, the degree of each vertex is finite. Hence, by König's lemma from graph theory, there is an infinite word $\omega$ such that $x \in X_{\left.\omega\right|_{n}}$ for all $n \geq 1$.

Remark. In view of (2.2), the map $\pi_{\Phi}$ is Hölder continuous if we endow $I^{\infty}$ with any of the standard metrics $\rho_{\alpha}, \alpha>0$, where

$$
\rho_{\alpha}(\omega, \tau)=\exp (-a \cdot|\omega \wedge \tau|)
$$

and $\omega \wedge \tau$ is the longest common initial segent of both $\omega$ and $\tau$.

2.5. Definition (Upper and lower pressure).

For any $t \geq 0$ and $n \in \mathbb{N}$, we define

$$
Z_{n}(t):=Z_{n}^{\Phi}(t):=\sum_{w \in I^{n}}\left\|D \varphi_{\omega}^{n}\right\|^{t}
$$

We now define the upper and lower pressure functions respectively as follows:

$$
\begin{aligned}
& \underline{P}(t):=\underline{P}^{\Phi}(t):=\liminf _{n \rightarrow \infty} \frac{1}{n} \log Z_{n} \quad \text { and } \\
& \bar{P}(t):=\bar{P}^{\Phi}(t):=\limsup _{n \rightarrow \infty} \frac{1}{n} \log Z_{n} .
\end{aligned}
$$

Note that $\underline{P}(t), \bar{P}(t) \in[-\infty,+\infty]$.

2.6. Lemma (Monotonicity). The lower pressure function is strictly decreasing when it is finite. That is, if $t_{1}<t_{2}$, then either both $\underline{P}\left(t_{1}\right)$ and $\underline{P}\left(t_{2}\right)$ are equal to $+\infty$, both are equal to $-\infty$, or $\underline{P}\left(t_{1}\right)>\underline{P}\left(t_{2}\right)$. The same is true of the upper pressure function.

Proof. Let $t \geq 0$ and $\varepsilon>0$. Then

$$
Z_{n}(t+\varepsilon)=\sum_{w \in I^{n}}\left\|D \varphi_{\omega}^{n}\right\|^{t+\varepsilon} \leq \sum_{w \in I^{n}} \eta^{n \varepsilon} \cdot\left\|D \varphi_{\omega}^{n}\right\|^{t}=\eta^{n \varepsilon} \cdot Z_{n}(t)
$$

where $\eta<1$ is the uniform contraction constant. So $\underline{P}(t+\varepsilon) \leq \underline{P}(t)-\varepsilon \cdot \log (1 / \eta)$. Hence, $\underline{P}(t+\varepsilon) \leq \underline{P}(t)$, and the inequality is strict if $\underline{P}(t)$ is finite.

The proof for the upper pressure function $\bar{P}$ is analogous.

We are now ready to define the Bowen dimension, our candidate for the Hausdorff dimension of $J(\Phi)$. 
2.7. Definition (Bowen dimension and Bowen's formula). Let $\Phi$ be a nonautonomous conformal iterated function system. We define

$$
h:=h(\Phi)=\operatorname{HD}(J(\Phi)) .
$$

The quantity

$$
\begin{aligned}
B:=B(\Phi) & :=\sup \{t \geq 0: \underline{P}(t)>0\}=\inf \{t \geq 0: \underline{P}(t)<0\} \\
& =\sup \left\{t \geq 0: Z_{n}(t) \rightarrow \infty\right\} .
\end{aligned}
$$

is called the Bowen dimension of the system $\Phi$. If the equality

$$
h(\Phi)=B(\varphi)
$$

is true, we say that Bowen's formula holds for $\Phi$.

Remark. The equalities stated in the definition of $B(\Phi)$ all follow from Lemma 2.6. Note that in the rather uninteresting case where $\underline{P}(0)=0$, the value of the two suprema should be taken to be zero by definition. Also note that $\underline{P}(t)<0$ for all $t>d$, as a consequence of the open set condition. Hence $B(\Phi) \leq d$.

The Bowen dimension is precisely the value that we obtain when restricting the covers in the definition of Hausdorff dimension to the "natural" covers

$$
\mathcal{V}_{n}:=\left\{X_{\omega}: \omega \in I^{n}\right\} .
$$

In particular, it is elementary that half of Bowen's formula is always satisfied:

2.8. Lemma. $h(\Phi) \leq B(\Phi)$.

We shall state and prove a more general technical statement (without increasing the difficulty of the proof), which will come for the discussion of counterexamples to Bowen's formula.

2.9. Lemma. Let $\Phi$ be a NCIFS. For each $n \geq 0$, let $\mathcal{U}_{n}$ be a covering of the limit set of the system $\Psi_{n}$ defined by $\Psi_{n}^{(j)}:=\Phi^{(n+j-1)}$, and denote its Hausdorff sum by

$$
S\left(\mathcal{U}_{n}, t\right):=\sum_{U \in \mathcal{U}_{n}} \operatorname{diam}(U)^{t} .
$$

Suppose that $t \geq 0$ is such that $\liminf _{n \rightarrow \infty} Z_{n-1}(t) \cdot S\left(\mathcal{U}_{n}, t\right)<\infty$.

Then $h(\Phi) \leq t$.

Remark. In our applications, $\mathcal{U}_{n}$ will usually be a covering of $\bigcup_{a \in I^{(n)}} \varphi_{a}^{(n)}(X) \supset J\left(\Psi_{n}\right)$.

Proof. Consider the covering

$$
\mathcal{V}_{n}:=\left\{\varphi_{\omega}(U): \omega \in I^{n-1}, U \in \mathcal{U}_{n}\right\}
$$

of $J(\Phi)$. Note that the diameters of the sets in $\mathcal{V}_{n}$ tend to zero as $n \rightarrow \infty$ by the uniform contraction assumption on $\Phi$.

Each set in this covering satisfies

$$
\operatorname{diam}\left(\varphi_{\omega}(U)\right) \leq C \cdot\left\|D \varphi_{\omega}\right\| \cdot \operatorname{diam}(U),
$$


where $C$ is a constant depending only on the geometry of $X$ 2. It follows that

$$
S\left(\mathcal{V}_{n}, t\right) \leq C \cdot\left(\sum_{\omega \in I^{n-1}}\left\|D \varphi_{\omega}\right\|^{t}\right) \cdot\left(\sum_{U \in \mathcal{U}_{n}} \operatorname{diam}(U)^{t}\right)=C \cdot Z_{n-1}(t) \cdot S\left(\mathcal{U}_{n}, t\right)
$$

Hence the assumption implies that the $t$-dimensional Hausdorff measure of $J(\Phi)$ is finite. In particular, $h(\Phi) \leq t$, as claimed.

Proof of Lemma 2.8. Let $t>B(\Phi)$, and let $\mathcal{U}_{n}=\{X\}$ for all $n$. We have

$$
\liminf _{n \rightarrow \infty} Z_{n}(t) \cdot S\left(\mathcal{U}_{n+1}, t\right)=\operatorname{diam}(X)^{t} \cdot \liminf _{n \rightarrow \infty} Z_{n}(t)=0
$$

by definition of $B(\Phi)$. Hence $h(\Phi) \leq t$ by the preceding lemma. Since $t>B(\Phi)$ was arbitrary, the claim follows.

To conclude the section, we remark that one cannot expect Bowen's formula $B(\Phi)=$ $J(\Phi)$ to hold without imposing any conditions on the non-autonomous system. Indeed, suppose that we are given a system $\Phi$ for which the upper pressure is strictly positive on the interval $[0, d)$, while the lower pressure is strictly negative on $(0, d]$.

(For example, let $\Phi_{1}$ be an autonomous system whose limit set has Hausdorff dimension $d$, such as the subdivision of the unit cube in $\mathbb{R}^{d}$ into $2^{d}$ cubes of side-length $1 / 2$, and let $\Phi_{2}$ be an autonomous system consisting of a single contraction, and whose limit set is hence a single point. If $0=N_{0}<N_{1}<\ldots$ is a sufficiently rapidly increasing sequence, then the system

$$
\Phi^{(k)}:= \begin{cases}\Phi_{1} & \text { if } N_{2 i}<k \leq N_{2 i+1} \text { for some } i \geq 0 \\ \Phi_{2} & \text { if } N_{2 i+1}<k \leq N_{2 i+2} \text { for some } i \geq 0\end{cases}
$$

has the desired properties.)

By Lemma 2.8, the limit set $J(\Phi)$ has Hausdorff dimension zero. Define $t_{k}:=d-1 / k$; by assumption on the upper pressure, there is an increasing sequence $n_{k}$ such that

$$
\liminf _{k \rightarrow \infty} \frac{1}{n_{k}} \log Z_{n_{k}}(d-1 / k) \geq 0
$$

We define a new system $\Psi$ by "collapsing" the levels between consecutive $n_{k}$. More precisely, $\Psi$ is given by index sets $J^{(k)}:=I^{n_{k}, n_{k+1}-1}$ and mappings

$$
\psi_{\omega}^{(k)}:=\varphi_{\omega}^{n_{k}, n_{k+1}-1} \quad\left(\omega \in J^{(k)}\right) .
$$

Assuming without loss of generality that $n_{1}=1$, this system has the same limit set $J(\Psi)=J(\Phi)$; so $h(\Psi)=h(\Phi)=0$. However, the lower pressure $\underline{P}^{\Psi}(t)$ is positive for every $t<d$, and hence $B(\Psi)=d$. Further counterexamples to Bowen's formula will be studied in Section 6 .

\footnotetext{
${ }^{2}$ If $X$ is convex, then $C=1$.
} 


\section{A LOWER BOUND FOR FINITE SYSTEMS}

In this section, we will prove a general lower bound on the Hausdorff dimension of $J(\Phi)$ for finite systems. Let us begin by making the following definition.

\subsection{Definition.}

A nonautonomous iterated function system is called finite if each alphabet $I^{(n)}, n \in \mathbb{N}$, is finite. It is called uniformly finite if there is a number $q \in \mathbb{N}$ such that $\# I^{(n)} \leq q$ for all $n \in \mathbb{N}$, and subexponentially bounded if

$$
\lim _{n \rightarrow \infty} \frac{1}{n} \log \# I^{(n)}=0 .
$$

Bowen's formula is based on the notion that the coverings of $J(\Phi)$ by sets of level $j$ (letting $j \rightarrow \infty$ ) provide the best coverings in terms of Hausdorff measure. As noted at the end of the previous section, we cannot expect this to be true without imposing extra conditions. One of the reasons for this is that, at some stages, the images of the maps in $\Phi^{(n)}$ might be grouped in such a way that a much more efficient covering is possible -imagine, in the one-dimensional case, that there is a very large number of tiny intervals, all of which are grouped together so that they can be covered by a single small interval. By the open set condition, the volume of

$$
\bigcup_{a \in I^{(n)}} \varphi_{a}^{(n)}(X)
$$

is (up to a constant factor) at least

$$
\# I^{(n)} \cdot \min _{a \in I^{(n)}}\left\|D \varphi_{a}^{(n)}\right\|^{d}
$$

So, if we set

$$
\underline{c}_{n}:=\min _{a \in I^{(n)}}\left\|D \varphi_{a}^{(n)}\right\|
$$

we have

$$
\operatorname{diam}\left(\bigcup_{a \in I^{(n)}} \varphi_{a}^{(n)}(X)\right) \geq \operatorname{const} \cdot \sqrt[d]{\# I^{(n)}} \cdot \underline{c}_{n} \cdot \operatorname{diam}(X) .
$$

In order to obtain a lower bound on the Hausdorff dimension, it thus makes sense to replace $Z_{n}(t)$ by

$$
\tilde{Z}_{n}(t):=Z_{n-1}(t) \cdot\left(\# I^{(n)}\right)^{\frac{t}{d}} \cdot \underline{c}_{n}^{t}
$$

in the definition of Bowen dimension.

We might expect that $\operatorname{HD}(J(\Phi)) \geq t$ provided that $\liminf \underline{Z}_{n}(t)>0$. The following result shows that this is indeed the case, provided that different pieces at the same level do not have drastically different sizes, as measured by the quantity

$$
\rho_{n}:=\sup _{a, b \in I^{(n)}} \frac{\left\|D \varphi_{a}^{(n)}\right\|}{\left\|D \varphi_{b}^{(n)}\right\|} \geq 1 .
$$


3.2. Theorem (Lower bounds on Hausdorff dimension). Let $\Phi$ be a finite nonautonomous conformal iterated function system. If $t \geq 0$ is such that

$$
\liminf _{n \rightarrow \infty} \frac{\tilde{Z}_{n}(t)}{1+\log \max _{j \leq n} \rho_{j}}>0
$$

then $\operatorname{HD}(J(\Phi)) \geq t$.

Proof. The proof will use the mass distribution principle, also known as the inverse Frostman lemma, compare [6, Section 4.1]. We begin by defining a sequence of probability measures $\left(m_{n}\right)_{n \in \mathbb{N}}$, with $m_{n}$ supported on the set $X_{n}$, as follows. For any $\omega \in I^{n}$, the restriction of $m_{n}$ to $X_{\omega}$ is a constant multiple of Lebesgue measure, chosen such that

$$
m_{n}\left(X_{\omega}\right)=\frac{\left\|D \varphi_{\omega}\right\|^{t}}{Z_{n}(t)} .
$$

Note that $\partial X$ has zero Lebesgue measure by the geometric assumption on $X$, and hence the set of points in $X_{n}$ that belong to several different sets $X_{\omega}$ also has zero Lebesgue measure.

Claim. Whenever $n \geq m$ and $\omega \in I^{m}$, we have

$$
m_{n}\left(X_{\omega}\right) \leq K^{t} \cdot \frac{\left\|D \varphi_{\omega}\right\|^{t}}{Z_{m}(t)}
$$

where $K$ is the bounded distortion constant.

Proof. Let $\tau \in I^{n}$ be an extension of a word $\omega^{\prime} \in I^{m}$. This means that there exists $\gamma \in I^{m+1, n}$ such that $\tau=\omega^{\prime} \gamma$. Then

$$
\left\|D \varphi_{\tau}\right\| \leq\left\|D \varphi_{\omega^{\prime}}\right\| \cdot\left\|D \varphi_{\gamma}^{m+1, n}\right\| \leq K \cdot\left\|D \varphi_{\tau}\right\| .
$$

by the bounded distortion condition. In particular,

$$
Z_{n}(t) \geq \frac{1}{K^{t}} \cdot Z_{m}(t) \cdot \sum_{\gamma \in I^{(m+1, n)}}\left\|D \varphi_{\gamma}^{m+1, n}\right\|^{t}
$$

and

$$
\begin{aligned}
m_{n}\left(X_{\omega}\right) & =\frac{\sum_{\gamma \in I^{m+1, n}}\left\|D \varphi_{\omega \gamma}\right\|^{t}}{Z_{n}(t)} \leq\left\|D \varphi_{\omega}\right\|^{t} \cdot \frac{\sum_{\gamma \in I^{m+1, n}}\left\|D \varphi_{\gamma}^{m+1, n}\right\|^{t}}{Z_{n}(t)} \\
& \leq\left\|D \varphi_{\omega}\right\|^{t} \cdot \frac{K^{t}}{Z_{m}(t)}
\end{aligned}
$$

Now fix $r>0$ and let $B$ be a ball in $\mathbb{R}^{d}$ of radius $r>0$. Consider the set $W$ of finite words $\omega$ such that:

- $B \cap\left(X_{\omega}\right) \neq \emptyset$;

- $\operatorname{diam}\left(X_{\omega}\right) \geq r$

- $\operatorname{diam}\left(X_{\omega a}\right) \leq r$ for some $a \in I^{(|w|+1)}$.

Let $W^{\prime}$ consist of those words in $W$ that are not extensions of some other word in $W$. Then the sets $X_{\omega}$, with $\omega \in W^{\prime}$, have pairwise disjoint interiors and diameter at least equal to $r$. By the uniform distortion property and the assumption on the geometry of $X$, there is a constant $M$, independent of $r$, such that no more than $M$ of the sets $X_{\omega}$, 
$\omega \in W$, can be pairwise disjoint. (The reason is that each set $X_{\omega}$ contains a cone of definite opening angle, based at a point of $B$ and of diameter comparable to $r$; see [9, Formula (2.10)]. The possible number of such cones that are pairwise disjoint must as we see by considering their total volume - necessarily be bounded independently of $r$. For details, compare [9, Lemma 2.7], which is stated for autonomous systems, but applies equally in our setting.)

In particular, $\# W^{\prime} \leq M$, and for every $k$, the number of words of length $k$ in $W$ is also bounded by $M$.

In the following, we use the term "const" to denote a positive constant that may depend on $\Phi$ and $t$, but not on the ball $B$ or the sets $W$ and $W^{\prime}$.

Fix $\tau \in W^{\prime}$ and put $k=|\tau|$. For $m \geq 0$, we define

$$
W_{\tau}(m):=\left\{\omega \in W \cap I^{k+m}: \tau=\left.\omega\right|_{k}\right\} .
$$

If $\omega \in W_{\tau}(m)$, then

$$
\operatorname{diam}\left(X_{\left.\omega\right|_{k+1}}\right) \leq \operatorname{const} \cdot r \cdot \rho_{k+1}
$$

and thus

$$
\operatorname{diam}\left(X_{\omega}\right) \leq \text { const } \cdot r \cdot \rho_{k+1} \cdot \eta^{m-1}
$$

by uniform contraction. Since $\operatorname{diam}\left(X_{\omega}\right)>r$ by definition of $W$, we see that $\rho_{k+1} \cdot \eta^{m}$ is bounded below by a positive constant. Thus

$$
m \leq \text { const } \cdot\left(1+\log \rho_{k+1}\right) .
$$

Since all words in $W_{\tau}(m)$ have length $k+m$, we must have $\# W_{\tau}(m) \leq M$, so we see that

$$
\begin{aligned}
& \# W_{\tau} \leq \text { const } \cdot\left(1+\log \rho_{|\tau|+1}\right), \quad \text { where } \\
& W_{\tau}=\bigcup_{m=0}^{\infty} W_{\tau}(m)=\bigcup\left\{W_{\tau}(m): 0 \leq m \leq \operatorname{const}\left(1+\log \rho_{|\tau|+1}\right)\right\}
\end{aligned}
$$

In particular,

$$
\Delta:=\max _{\omega \in W}|\omega|<\infty .
$$

Now let $\omega \in W$ and set $n:=|\omega|$. Let $A_{\omega} \subset I^{(n+1)}$ be the set of all $a \in I^{(n+1)}$ for which $X_{\omega a}$ intersects $B$ and $\operatorname{diam}\left(X_{\omega a}\right) \leq r$. Then

$$
B \cap J(\Phi) \subset \bigcup_{w \in W} \bigcup_{a \in A_{\omega}} X_{\omega a}
$$

and the sets in the union on the right hand side have pairwise disjoint interiors. Since the disk of radius $2 r$ and with the same center as $B$ contains $\bigcup_{a \in A_{\omega}} X_{\omega a}$, we have

$$
r^{d} \geq \mathrm{const} \cdot \sum_{i \in A_{\omega}} \operatorname{diam}\left(X_{\omega i}\right)^{d} \geq \mathrm{const} \cdot\left\|D \varphi_{\omega}\right\|^{d} \cdot \sum_{a \in A_{\omega}}\left\|D \varphi_{a}^{(n+1)}\right\|^{d} .
$$

Let $q \geq \Delta+1$. We are going to estimate the measure of the inner union in (3.7) with respect to the measure $m_{q}$. First note that that using (3.4) and the definition of $\Delta$, we 
get from (3.4) that

$$
\begin{aligned}
m_{q}\left(\bigcup_{a \in A_{\omega}} X_{\omega a}\right) & \leq \sum_{a \in A_{\omega}} m_{q}\left(X_{\omega a}\right) \leq \frac{K^{t}}{Z_{n+1}(t)} \sum_{a \in A_{\omega}}\left\|D \varphi_{\omega a}\right\|^{t} \\
& \leq \frac{K^{t} \cdot\left\|D \varphi_{\omega}\right\|^{t}}{Z_{n+1}(t)} \sum_{a \in A_{\omega}}\left\|D \varphi_{a}^{(n+1)}\right\|^{t} .
\end{aligned}
$$

By (3.8), we can estimate

$$
\left\|D \varphi_{\omega}\right\| \leq \mathrm{const} \cdot \frac{r}{\sqrt[d]{\sum_{a \in A_{\omega}}\left\|D \varphi_{a}^{(n+1)}\right\|^{d}}}
$$

SO

$$
m_{q}\left(\bigcup_{a \in A_{\omega}} X_{\omega a}\right) \leq \frac{\text { const } \cdot r^{t}}{Z_{n+1}(t)} \cdot \frac{\sum_{a \in A_{\omega}}\left\|D \varphi_{a}^{(n+1)}\right\|^{t}}{\left(\sum_{a \in A_{\omega}}\left\|D \varphi_{a}^{(n+1)}\right\|^{d}\right)^{t / d}}
$$

The fraction on the right can be estimated using Hölder's inequality: putting $\vartheta:=d / t \geq$ 1 , we see that

$$
\frac{\sum_{a \in A_{\omega}}\left\|D \varphi_{a}^{(n+1)}\right\|^{t}}{\left(\sum_{a \in A_{\omega}}\left\|D \varphi_{a}^{(n+1)}\right\|^{d}\right)^{t / d}}=\frac{\sum_{a \in A_{\omega}}\left\|D \varphi_{a}^{(n+1)}\right\|^{t}}{\left(\sum_{a \in A_{\omega}}\left(\left\|D \varphi_{a}^{(n+1)}\right\|^{t}\right)^{\vartheta}\right)^{1 / \vartheta}} \leq\left(\# A_{\omega}\right)^{1-1 / \vartheta} \leq\left(\# I^{(n+1)}\right)^{1-t / d} .
$$

So

$$
m_{q}\left(\bigcup_{a \in A_{\omega}} X_{\omega a}\right) \leq \frac{\mathrm{const} \cdot r^{t}}{Z_{n+1}(t)} \cdot\left(\# I^{(n+1)}\right)^{1-t / d}
$$

Now $Z_{n+1}(t) \geq \mathrm{const} \cdot Z_{n}(t) \cdot \# I^{(n+1)} \cdot \underline{c}_{n+1}^{t}$, and therefore

$$
\begin{aligned}
m_{q}\left(\bigcup_{a \in A_{\omega}} X_{\omega a}\right) & \leq \frac{\text { const } \cdot r^{t}}{Z_{n+1}(t)} \cdot\left(\# I^{(n+1)}\right)^{1-t / d} \\
& \leq \frac{\mathrm{const}}{Z_{n}(t) \cdot\left(\# I^{(n+1)}\right)^{t / d} \cdot \underline{c}_{n+1}^{t}}=\frac{\text { const }}{\tilde{Z}_{n+1}(t)} \cdot r^{t} .
\end{aligned}
$$

Now let $\tau \in W^{\prime}$, and $\omega \in W_{\tau}$. Then, by the assumption of the theorem, we get

$$
\begin{aligned}
m_{q}\left(\bigcup_{a \in A_{\omega}} X_{\omega a}\right) & \leq \frac{\text { const }}{\widetilde{Z}_{n+1}(t)} \cdot r^{t} \leq \frac{\text { const }}{1+\log \max _{j \leq n+1} \rho_{j}} \cdot r^{t} \\
& \leq \frac{\text { const }}{1+\log \rho_{|\tau|+1}} \cdot r^{t}
\end{aligned}
$$


provided that $r$ was chosen sufficiently small, and hence $n=|\omega|$ is sufficiently large. So we can apply (3.5) to see that

$$
\begin{aligned}
m_{q}(B) & \leq m_{q}\left(\bigcup_{\tau \in W^{\prime}} \bigcup_{\omega \in W_{\tau}} \bigcup_{a \in A_{\omega}} X_{\omega a}\right) \leq \sum_{\tau \in W^{\prime}} \sum_{\omega \in W_{\tau}} m_{q}\left(\bigcup_{a \in A_{\omega}} X_{\omega a}\right) \\
& \leq \sum_{\tau \in W^{\prime}} r^{t} \sum_{\omega \in W_{\tau}} \frac{\operatorname{const}}{1+\log \rho_{|\tau|+1}} \\
& \leq \operatorname{const} r^{t} \sum_{\tau \in W^{\prime}} \frac{\# W_{\tau}}{1+\log \rho_{|\tau|+1}} \\
& \leq \operatorname{const} r^{t} .
\end{aligned}
$$

Hence, if $m$ is an arbitrary weak limit of the sequence $\left(m_{q}\right)_{1}^{\infty}$, then

$$
m(B) \leq \text { const } r^{t}
$$

and, by the Converse Frostman Lemma, it follows that $\operatorname{HD}(J(\Phi)) \geq t$, as claimed.

Remark. It is tempting to replace the rather coarse bound in the definition of $\tilde{Z}_{n}(t)$, which uses the minimal size of a piece at level $n$, by

$$
\sqrt[d]{\sum_{a \in I^{(n)}}\left\|D \varphi_{a}^{(n)}\right\|^{d}}
$$

Unfortunately, with this alternative definition, Theorem 3.2 no longer holds. We shall leave it to the reader to construct a counterexample, which can be done along similar lines as in Section 6.

However, in view of (3.9) we can replace $\tilde{Z}_{n}(t)$ in the statement of the theorem by

$$
\hat{Z}_{n}(t):=Z_{n}(t) \cdot \min _{A \subset I^{(n)}} \frac{\sum_{a \in A}\left(\left\|D \varphi_{a}^{(n)}\right\|^{d}\right)^{t / d}}{\sum_{a \in A}\left\|D \varphi_{a}^{(n)}\right\|^{t}} .
$$

We shall revisit this idea in the proof of Theorem 4.6.

\section{BALANCING CONDITIONS}

We now use Theorem 3.2 to establish Bowen's formula in certain cases. In particular, we shall prove Corollary 4.3, an important special case of Theorem 1.1 that is essential for the proof of the full theorem.

We need to make assumptions on how much the sizes of the different sets (as measured by the norm of derivatives) vary at a fixed level $n$; i.e., we need to bound the quantity $\rho_{n}$ that was defined in (3.3):

$$
\rho_{n}:=\sup _{a, b \in I^{(n)}} \frac{\left\|D \varphi_{a}^{(n)}\right\|}{\left\|D \varphi_{b}^{(n)}\right\|} \geq 1 .
$$

We note, however, that, in the next section, we will be able to remove the hypotheses on $\rho_{n}$ from many of the results we prove here. 
4.1. Definition (Balancing conditions).

A non-autonomous conformal iterated function system is called perfectly balanced if $\rho_{n}=1$ for all $n$ and furthermore all $\varphi_{i}^{(n)}$ are affine similarities.

The system is called balanced if there is a constant $\kappa \geq 1$ such that $\rho_{n} \leq \kappa$ for all $n \in \mathbb{N}$, and weakly balanced if

$$
\lim _{n \rightarrow \infty} \frac{1}{n} \log \rho_{n}=0 .
$$

Finally, we call $\Phi$ barely balanced if

$$
\lim _{n \rightarrow \infty} \frac{\log \left(1+\log \rho_{n}\right)}{n}=0 .
$$

For the purposes of the estimates on Hausdorff dimension in this paper, we shall see that weakly balanced systems do not differ from perfectly balanced ones. That is, all our positive results apply to weakly balanced systems, while all weakly-balanced counterexamples can be constructed to be perfectly balanced.

The "barely balanced" condition is of importance mainly because, for these systems, Theorem 3.2 becomes a lower bound depending only on the behavior of the functions $\tilde{Z}_{n}(t)$. Recall that this quantity was defined in (3.2) as

$$
\tilde{Z}_{n}(t):=Z_{n-1}(t) \cdot\left(\# I^{(n)}\right)^{\frac{t}{d}} \cdot \underline{c}_{n}^{t}
$$

4.2. Lemma (Lower bound for barely balanced systems). Let $\Phi$ be a finite NCIFS.

(a) The function

$$
\tilde{P}(t):=\liminf _{n \rightarrow \infty} \frac{1}{n} \log \tilde{Z}_{n}(t)
$$

is strictly decreasing when it is finite; in fact,

$$
\tilde{P}\left(t^{\prime}\right) \geq \tilde{P}(t)+\left(t-t^{\prime}\right) \cdot \log \frac{1}{\eta}
$$

when $t^{\prime}<t$ and $\tilde{P}(t)$ is finite. (Here $\eta$ denotes the contraction constant, as usual.)

(b) Suppose that $\Phi$ is barely balanced, and that $t \geq 0$ is such that

$$
\tilde{P}(t):=\liminf _{n \rightarrow \infty} \frac{1}{n} \log \tilde{Z}_{n}(t) \geq 0 .
$$

Then $\operatorname{HD}(J(\Phi)) \geq t$.

Proof. By definition of $\underline{c}_{n}$ and Bounded Distortion, the image of each $\varphi_{i}^{(n)}$ contains a ball of radius comparable to $\underline{c}_{n}$. By the Open Set Condition, it follows that there exists a constant $C>0$ such that

$$
\# I^{(n+1)} \cdot \underline{c}_{n+1}^{d} \leq C
$$

for all $n \geq 0$. Hence, for $t^{\prime}<t$,

$$
\frac{\tilde{Z}_{n+1}(t)}{\tilde{Z}_{n+1}\left(t^{\prime}\right)}=\frac{Z_{n}(t)}{Z_{n}\left(t^{\prime}\right)}\left(\left(\# I^{(n+1)}\right)^{1 / d} \underline{c}_{n+1}\right)^{t-t^{\prime}} \leq \frac{Z_{n}(t)}{Z_{n}\left(t^{\prime}\right)} C^{\frac{1}{d}\left(t-t^{\prime}\right)} \leq C^{\frac{1}{d}\left(t-t^{\prime}\right)} \eta^{\left(t-t^{\prime}\right) n}
$$


for sufficiently large $n$. So

$$
\begin{aligned}
\tilde{P}\left(t^{\prime}\right)=\liminf _{n \rightarrow \infty} \frac{1}{n+1} \tilde{Z}_{n+1}\left(t^{\prime}\right) & \geq\left(t-t^{\prime}\right) \cdot \log (1 / \eta)+\liminf _{n \rightarrow \infty}\left(\frac{\tilde{Z}_{n}(t)}{n}-\frac{\left(t-t^{\prime}\right) \log C}{d n}\right) \\
& =\left(t-t^{\prime}\right) \cdot \log (1 / \eta)+\tilde{P}(t) .
\end{aligned}
$$

This proves the first claim. Now suppose that $\Phi$ is barely balanced and that $\tilde{P}(t) \geq 0$. Let $t^{\prime}<t$ be arbitrary. Then $\tilde{P}\left(t^{\prime}\right)>0$, so $\tilde{Z}_{n}\left(t^{\prime}\right)$ grows at least exponentially in $n$, while the denominator in the hypothesis of Theorem 3.2 grows subexponentially since $\Phi$ is barely balanced. So $\operatorname{HD}(J(\Phi)) \geq t^{\prime}$ by Theorem 3.2. Since $t^{\prime}<t$ was arbitrary, we have obtained the desired conclusion.

For the remainder of the section, we shall study weakly balanced systems.

4.3. Corollary (Bowen's formula for weakly balanced systems). Let $\Phi$ be a nonautonomous iterated function system that is subexponentially bounded and weakly balanced. Then $\operatorname{HD}(J(\Phi))=B(\Phi)$.

Proof. Let $t<B(\Phi)$. Then $\underline{P}(t)>0$, and hence $Z_{n}(t)$ grows at least exponentially in $n$. We have

$$
\begin{aligned}
Z_{n+1}(t) \leq Z_{n}(t) \cdot \sum_{a \in I^{(n+1)}}\left\|D \varphi_{a}^{(n+1)}\right\|^{t} & \leq Z_{n}(t) \cdot \# I^{(n+1)} \cdot\left(\rho_{n+1} \cdot \underline{c}_{n+1}\right)^{t} \\
& =\tilde{Z}_{n+1}(t) \cdot\left(\# I^{n+1}\right)^{1-t / d} \cdot \rho_{n+1}^{t} .
\end{aligned}
$$

Since the system $\Phi$ is subexponentially bounded and weakly balanced, $\left(\# I^{(n+1)}\right)^{1-t / d} \rho_{n+1}^{t}$ grows at most subexponentially in $n$. So

$$
\liminf _{n \rightarrow \infty} \frac{1}{n} \log \tilde{Z}_{n}(t) \geq \liminf _{n \rightarrow \infty} \frac{1}{n} Z_{n}(t)=\underline{P}(t)>0 .
$$

Corollary 4.2 implies that $\operatorname{HD}(J(\Phi)) \geq t$.

Since $t<B(\Phi)$ was arbitrary, we see that $\operatorname{HD}(J(\Phi)) \geq B(\Phi)$. Together with Lemma 2.8, this completes the proof.

As we shall see in the next section, the condition that $\Phi$ is subexponentially bounded cannot be relaxed: there are perfectly balanced systems where Bowen's formula fails and $\# I^{(n)}$ has arbitrarily small exponential growth.

However, for these examples the contraction constants, along with the numbers \# $I^{(n)}$, behave very irregularly: for many values of $n, \bar{c}_{n}$ is bounded away from zero and \# $I^{(n)}$ is finite. The next result, another interesting consequence of Theorem 3.2, establishes part of Theorem 1.2 for (sufficiently balanced) systems of at most exponential growth when $\bar{c}_{n} \rightarrow 0$.

4.4. Corollary (Systems of exponential growth). Suppose that $\Phi$ is a nonautonomous conformal IFS such that

$$
\limsup _{n \rightarrow \infty} \frac{1}{n} \log \# I^{(n)}<\infty \quad \text { and } \quad \limsup _{n \rightarrow \infty} \frac{1}{n} \log \rho_{n}<\infty .
$$

If $\lim _{n \rightarrow \infty} \bar{c}_{n}=0$, then Bowen's formula holds. (Recall that $\bar{c}_{n}=\underline{c}_{n} \cdot \rho_{n}$ is the largest derivative of a map in $\Phi^{(n)}$.) 
Proof. The key observation is that $Z_{n}(t)$ grows superexponentially for $t<B(\Phi)$, which is enough to show that $\tilde{P}(t)>0$.

Indeed, fix $t<B(\Phi)$ and let $\varepsilon>0$ and $t^{\prime}:=t-\varepsilon$. For large $n$, we have

$$
Z_{n}\left(t^{\prime}\right)=\sum_{\omega \in I^{n}}\left\|D \varphi_{\omega}^{n}\right\|^{t-\varepsilon} \geq Z_{n}(t) \cdot\left(\max _{\omega \in I^{n}}\left\|D \varphi_{\omega}^{n}\right\|\right)^{-\varepsilon} \geq Z_{n}(t) \cdot \prod_{j=1}^{n} \bar{c}_{j}^{-\varepsilon} \geq \prod_{j=1}^{n} \bar{c}_{j}^{-\varepsilon} .
$$

(Here $K$ is the distortion constant.) Since $\bar{c}_{n} \rightarrow 0$, we have

$$
\frac{\sum_{j=1}^{n}-\log \bar{c}_{n}}{n} \rightarrow \infty
$$

and hence $\left(\log Z_{n}(t-\varepsilon)\right) / n \rightarrow \infty$. As in the preceding proof, we have

$$
\tilde{Z}_{n}\left(t^{\prime}\right) \geq \frac{Z_{n}\left(t^{\prime}\right)}{\left(\# I^{(n)}\right)^{1-t^{\prime} / d} \cdot \rho_{n}^{t^{\prime}}}
$$

We just saw that the numerator in this expression grows superexponentially, while the denominator grows at most exponentially fast by assumption. By Lemma 4.2, it follows that $\operatorname{HD}(J(\Phi)) \geq t-\varepsilon$. As $t<B(\Phi)$ and $\varepsilon>0$ were arbitrary, we see that $\operatorname{HD}(J(\Phi)) \geq$ $B(\Phi)$, as desired.

Remark 1 . The requirement that $\bar{c}_{n} \rightarrow 0$ is satisfied whenever $\Phi$ is weakly balanced and $\lim \inf \left(\log \# I^{(n)}\right) / n>0$.

Remark 2. The proof shows that the assumption $\bar{c}_{n} \rightarrow 0$ can be replaced by

$$
\frac{\sum_{j=1}^{n} \log \bar{c}_{n}}{n} \rightarrow \infty
$$

In a similar vein, we can now prove Proposition 1.3, which shows that we can explicitly calculate the Hausdorff dimension whenever both the number of pieces at each level and their sizes have regular exponential growth. Using our notation from this section, we can restate the result as follows.

4.5. Corollary. Suppose that $\Phi$ is weakly balanced, and that furthermore both limits

$$
a:=\lim _{n \rightarrow \infty} \frac{1}{n} \log \# I^{(n)}
$$

and

$$
b:=\lim _{n \rightarrow \infty} \frac{1}{n} \log \left(1 / \bar{c}_{n}\right)
$$

exist and are finite and positive. Then $\operatorname{HD}(J(\Phi))=B(\Phi)=a / b$.

Proof. The previous Corollary implies that Bowen's formula holds, hence it remains to show that $B(\Phi)=a / b$.

Because $\Phi$ is weakly balanced, we have

$$
b=\lim _{n \rightarrow \infty} \frac{1}{n} \log \left(1 / \underline{c}_{n}\right) .
$$

For any $t$, we have

$$
Z_{n+1}(t) \leq Z_{n}(t) \cdot \# I^{(n+1)} \cdot \bar{c}_{n+1}^{t}
$$


and

So

$$
Z_{n+1}(t) \geq \mathrm{const} \cdot Z_{n}(t) \cdot \# I^{(n+1)} \cdot \underline{c}_{n+1}^{t}
$$

$$
\text { const } \cdot \# I^{(n+1)} \cdot \underline{c}_{n+1}^{t} \leq \frac{Z_{n+1}(t)}{Z_{n}(t)} \leq \# I^{(n+1)} \cdot \bar{c}_{n+1}^{t} .
$$

By assumption, if $t>a / b$, the right-hand side tends to zero as $n \rightarrow \infty$. Likewise, for $t<a / b$, the left hand side tends to $\infty$. This implies that $Z_{n}(t)$ tends to 0 for $t>a / b$ and to $\infty$ for $t<a / b$, and hence $B(\Phi)=a / b$, as claimed.

Remark. The proof above gives a litttle bit more. Namely, suppose that $\Phi$ is a nonautonomous conformal IFS such that

$$
0<a_{-}:=\liminf _{n \rightarrow \infty} \frac{\log \# I^{(n)}}{n} \leq \limsup _{n \rightarrow \infty} \frac{\log \# I^{(n)}}{n}=: a_{+}<\infty
$$

and

$$
0<b_{-}:=\liminf _{n \rightarrow \infty} \frac{\log \left(1 / \bar{c}_{n}\right)}{n} \leq \limsup _{n \rightarrow \infty} \frac{\log \left(1 / \underline{c}_{n}\right)}{n}=: b_{+}<\infty
$$

then

$$
\frac{a_{-}}{b_{+}} \leq \mathrm{HD}(J(\Phi)) \leq \frac{a_{+}}{b_{-}}
$$

Together with Corollary 4.4, the following result provides a preliminary version of Theorem 1.2 for sufficiently balanced systems of at most exponential growth.

4.6. Theorem (Systems with extremal Hausdorff and Bowen dimension). Let $\Phi$ be a finite non-autonomous conformal iterated function system such that

$$
\limsup _{n \rightarrow \infty} \frac{\log \# I^{(n)}}{n}<\infty \quad \text { and } \limsup \log \rho_{n} / n<\infty .
$$

If $\operatorname{HD}(J(\Phi))=0$, then $B(\Phi)=0$, and if $B(\Phi)=d$, then $\operatorname{HD}(J(\Phi))=d$.

Proof. For every $t \geq 0$, we again consider

$$
\tilde{P}(t)=\liminf _{n \rightarrow \infty} \frac{1}{n} \log \tilde{Z}_{n}(t) .
$$

Let us also set

$$
\underline{a}=\liminf _{n \rightarrow \infty} \frac{\log \# I^{(n)}}{n} \geq 0, \quad \text { and } \quad \bar{a}=\limsup _{n \rightarrow \infty} \frac{\log \# I^{(n)}}{n}<\infty .
$$

For the first implication of the theorem, let us suppose that $B(\Phi)>0$, and that $\Phi$ is not necessarily weakly bounded, but that

$$
\bar{\rho}:=\limsup _{n \rightarrow \infty} \frac{\log \rho_{n}}{n}<\infty .
$$

We must show that $\operatorname{HD}(J(\Phi))>0$. To do fix $0<t_{0}<B(\Phi)$, so that $\underline{P}\left(t_{0}\right)>0$. Recall that

It follows that

$$
Z_{n+1}\left(t_{0}\right) \leq Z_{n}\left(t_{0}\right) \cdot \# I^{(n+1)} \cdot \bar{c}_{n}^{t_{0}}=Z_{n}\left(t_{0}\right) \cdot \# I^{(n+1)} \cdot \rho_{n}^{t_{0}} \cdot \underline{c}_{n}^{t_{0}} .
$$

$$
\liminf _{n \rightarrow \infty} \frac{\log Z_{n}\left(t_{0}\right)+t_{0} \cdot \log \underline{c}_{n}}{n} \geq \underline{P}\left(t_{0}\right)-\bar{a}-t_{0} \cdot \bar{\rho}>-\infty .
$$


Now let $t \in\left(0, t_{0}\right)$, and observe that

$$
\begin{aligned}
\log \tilde{Z}_{n+1}(t) & =\log Z_{n}(t)+\frac{t}{d} \log \# I^{(n+1)}+t \cdot \log \underline{c}_{n} \\
& >\log Z_{n}\left(t_{0}\right)+\frac{t}{d} \log \# I^{(n+1)}+t \cdot \log \underline{c}_{n} \\
& =\left(1-\frac{t}{t_{0}}\right) \cdot \log Z_{n}\left(t_{0}\right)+\frac{t}{d} \log \# I^{(n+1)}+\frac{t}{t_{0}} \cdot\left(\log Z_{n}\left(t_{0}\right)+t_{0} \cdot \log \underline{c}_{n}\right) .
\end{aligned}
$$

Thus

$$
\tilde{P}(t) \geq\left(1-\frac{t}{t_{0}}\right) \cdot \underline{P}\left(t_{0}\right)+\frac{t}{d} \cdot \underline{a}+\frac{t}{t_{0}} \cdot\left(\underline{P}\left(t_{0}\right)-\bar{a}-\bar{\rho}\right) .
$$

The right hands side tends to $\underline{P}\left(t_{0}\right)$ as $t \rightarrow 0$; in particular, $\tilde{P}(t)$ is positive for sufficiently small positive $t$. Hence $\operatorname{HD}(J(\Phi)) \geq t>0$, as claimed.

For the second implication, suppose that $B(\Phi)=d$. Let $\tilde{t}<d$, and choose $t \in(\tilde{t}, d)$ sufficiently close to $d$, as indicated below. We recall the proof of Theorem 3.2. Let $m$ be the measure constructed there, let $B$ again be a ball of radius $r>0$, and continue the proof analogously up to (3.10):

$$
m\left(\bigcup_{a \in A_{\omega}} X_{\omega a}\right) \leq \frac{\mathrm{const} \cdot r^{t}}{Z_{n+1}(t)} \cdot\left(\# I^{(n+1)}\right)^{1-t / d} .
$$

Recall that $\# I^{(n+1)}$ grows at most exponentially fast. Also recall that $n=|\omega|$, where $\omega$ was such that

$$
r \leq \operatorname{diam}\left(X_{\omega}\right) \leq \mathrm{const} \cdot\left\|D \varphi_{\omega}\right\| \leq \mathrm{const} \cdot \eta^{n}
$$

Hence

$$
m\left(\bigcup_{a \in A_{\omega}} X_{\omega a}\right) \leq \frac{\mathrm{const} \cdot r^{\tilde{t}}}{Z_{n+1}(t)} \cdot r^{t-\tilde{t}} \cdot\left(\# I^{(n+1)}\right)^{1-t / d} \leq \frac{\mathrm{const} \cdot r^{\tilde{t}}}{Z_{n+1}(t)} \cdot \eta^{n(t-\tilde{t})} \cdot\left(\# I^{(n+1)}\right)^{1-t / d} .
$$

If $t$ was chosen sufficiently close to $d$ (depending on $\eta$, the exponential growth rate of $I^{(n+1)}$ and $\left.\tilde{t}\right)$, the second term in the product tends to zero faster than the final term tends to infinity. Hence we have, for large $n$,

$$
m\left(\bigcup_{a \in A_{\omega}} X_{\omega a}\right) \leq \frac{\text { const } \cdot r^{\tilde{t}}}{Z_{n+1}(t)}
$$

Now let $\tau \in W^{\prime}$ and $\omega \in W_{\tau}$. Since $\rho_{n}$ grows at most exponentially, and $Z_{n}(t)$ grows at least exponentially, we see that

$$
m\left(\bigcup_{a \in A_{\omega}} X_{\omega a}\right) \leq \frac{\mathrm{const} \cdot r^{\tilde{t}}}{Z_{n+1}(t)} \leq \frac{\mathrm{const}}{1+\log \rho_{|\tau|+1}} \cdot r^{\tilde{t}}
$$

We now continue as in the proof of Theorem 3.2 , and see that $\operatorname{HD}(J(\Phi)) \geq \tilde{t}$. Since $\tilde{t}<d$ was arbitrary, we are done. 


\section{Approximation By subsystems}

In this section, we shall study the approximation of a nonautonomous system $\Phi$ (with finite or infinite alphabets) by suitably chosen finite subsystems. The purpose of this procedure is two-fold. On the one hand, we will be able to remove the balancing assumptions in many of the results of the preceding sections. Indeed, essentially we will show that we can always restrict to a subsystem that does satisfy these assumptions, and whose pressure function is close to the original one. In particular, we complete the proof of Theorem 1.1 in this section. On the other hand, the methods developed are crucial for our study of infinite systems in the second half of the paper.

The key result is as follows.

5.1. Proposition (Approximation by finite subsystems). Consider a (possibly infinite) non-autonomous iterated functions system $\Phi$. Let $t \in[0, d]$ and assume that

$$
\sum_{j \in I^{(n)}}\left\|D \varphi_{j}\right\|^{t}<\infty
$$

for all $n$. Let $\delta>0$, and, for each $n$, let $I_{f}^{(n)} \subset I^{(n)}$ be finite such that

$$
\sum_{j \in I^{(n)}}\left\|D \varphi_{j}\right\|^{t} \leq(1+\delta) \sum_{j \in I_{f}^{(n)}}\left\|D \varphi_{j}\right\|^{t}
$$

for all sufficiently large $n$.

If $\Phi_{f}$ is the system obtained by using $I_{f}^{(n)}$ as index sets instead of $I^{(n)}$, then

$$
\underline{P}_{f}(t):=\underline{P}^{\Phi_{f}}(t) \geq \underline{P}(t)-\delta \cdot K^{2 d} .
$$

Proof. We may assume without loss of generality that (5.2) holds for all $n$. If the system $\Phi$ consists of affine similarities, then the result is immediate from the definitions. In the case where the maps are nonlinear, the idea is the same, but we need to deal with the distortion constants. To do so, we must introduce notation that allows us to decompose a word $\omega$ into those symbols that belong to the "finite" parts $I_{f}^{(n)}$ of the index sets, and those that do not. Let us fix $n \in \mathbb{N}$ in the following.

For $n \geq 1$ and $0 \leq s \leq n$, let $\mathcal{P}_{s}$ be the collection of all subsets of $\mathbb{N}_{n}=\{1, \ldots, n\}$ having exactly $s$ elements. Given $P \in \mathcal{P}_{s}$, we denote by $I^{P}$ the set of all "words" $\left(\omega_{j}\right)_{j \in P}$ with $\omega_{j} \in I^{(j)}$ for all $j \in P$, and similarly

$$
I_{f}^{P}:=\left\{\left(\omega_{j}\right)_{j \in P}: \omega_{j} \in I_{f}^{(j)}\right\} ; \quad I_{\infty}^{P}:=\left\{\left(\omega_{j}\right)_{j \in P}: \omega_{j} \in I^{(j)} \backslash I_{f}^{(j)}\right\} .
$$

Now fix $s \in\{0, \ldots, n\}$ and $P \in \mathcal{P}_{s}$. We can combine two words $\omega \in I^{P}$ and $\tau \in I^{\mathbb{N}_{n} \backslash P}$ to a conventional word of length $n$, which we denote $\omega \star \tau \in I^{n}$. More precisely,

$$
(\omega \star \tau)_{j}= \begin{cases}\omega_{j} & \text { if } j \in P \\ \tau_{j} & \text { if } j \notin P . .\end{cases}
$$

Let $k \leq n+1-s$ be the number of maximal continuous segments of integers in $P$. Then one can think of $\omega \in I^{P}$ as consisting of $k$ separate words $\omega^{1}, \ldots, \omega^{k}$, with 
$\omega^{i} \in I^{m_{i}, n_{i}}$ (i.e., $\omega^{i}$ starts at index $m_{i}$ and ends at index $n_{i}$ ), where $m_{i} \leq n_{i}<m_{i+1}$. We abbreviate

$$
\left\|D \varphi_{\omega}^{(P)}\right\|:=\prod_{i=1}^{k}\left\|D \varphi_{\omega^{i}}^{m_{i}, n_{i}}\right\|
$$

For any $\tau \in I^{\mathbb{N}_{n} \backslash P}$, we have

$$
\left\|D \varphi_{\omega \star \tau}\right\| \leq\left\|D \varphi_{\omega}^{(P)}\right\| \cdot \prod_{j \in \mathbb{N}_{n} \backslash P}\left\|D \varphi_{\tau_{j}}^{(j)}\right\| \leq K^{k+n-s-1} \cdot\left\|D \varphi_{\omega \star \tau}\right\| \leq K^{2(n-s)} \cdot\left\|D \varphi_{\omega \star \tau}\right\| .
$$

Note that here we split the word $\omega \star \tau$ into $k+n-s$ pieces, each of which is one of the words $\omega^{i}$ or one of the symbols of $\tau$. Hence, in the second inequality, we applied the bounded distortion property precisely $k+n-s-1$ times, resulting in the stated factor.

With these preparations, we can now estimate, for each $P$, the contribution $Z_{n}^{(P)}(t)$ to the sum $Z_{n}(t)$ of the words having exactly $s$ entries in $I_{f}^{P}$, in the positions prescribed by $P$. We begin by splitting each of these words as in (5.31) and rearranging:

$$
\begin{aligned}
Z_{n}^{(P)}(t):=\sum_{(\omega, \tau) \in I_{f}^{P} \times I_{\infty}^{\mathbb{N}_{n} \backslash P}}\left\|D \varphi_{\omega \star \tau}\right\|^{t} & \leq \sum_{\omega \in I_{f}^{P}}\left\|D \varphi_{\omega}^{(P)}\right\|^{t} \sum_{\tau \in I_{\infty}^{\mathbb{N}_{n} \backslash P}} \prod_{j \in \mathbb{N}_{n} \backslash P}\left\|D \varphi_{\tau_{j}}^{(j)}\right\|^{t} \\
& =\sum_{\omega \in I_{f}^{P}}\left\|D \varphi_{\omega}^{(P)}\right\|^{t} \prod_{j \in \mathbb{N}_{n} \backslash P} \sum_{a \in I^{(j)} \backslash I_{f}^{(j)}}\left\|D \varphi_{a}^{(j)}\right\|^{t} .
\end{aligned}
$$

By assumption, we can estimate the final sum as follows:

$$
\begin{aligned}
Z_{n}^{(P)}(t) & \leq \sum_{\omega \in I_{f}^{P}}\left\|D \varphi_{\omega}^{(P)}\right\|^{t} \prod_{j \in \mathbb{N}_{n} \backslash P} \delta \sum_{a \in I_{f}^{(j)}}\left\|D \varphi_{a}^{(j)}\right\|^{t} \\
& =\delta^{n-s} \sum_{\omega \in I_{f}^{P}}\left\|D \varphi_{\omega}^{(P)}\right\|^{t} \prod_{j \in \mathbb{N}_{n} \backslash P} \sum_{a \in I_{f}^{(j)}}\left\|D \varphi_{a}^{(j)}\right\|^{t} .
\end{aligned}
$$

Finally, we apply the second inequality of (5.3) to recombine the words:

$$
Z_{n}^{(P)}(t) \leq \delta^{(n-s)} K^{2 t(n-s)} \sum_{\omega \in I_{f}^{\mathbb{N}_{n}}}\left\|D \varphi_{\omega}\right\|^{t}=\delta^{n-s} K^{2 t(n-s)} Z_{n}^{\Phi_{f}}(t) .
$$

Thus we can bound the sum $Z_{n}(t)$, by summing over all possible sets $P$ :

$$
\begin{aligned}
Z_{n}(t) & =\sum_{|\omega|=n}\left\|D \varphi_{\omega}\right\|^{t}=\sum_{s=0}^{n} \sum_{P \in \mathcal{P}_{s}} \sum_{(\omega, \tau) \in I_{f}^{P} \times I_{\infty}^{\mathbb{N}_{n} \backslash P}}\left\|D \varphi_{\omega \star \tau}\right\|^{t} \\
& \leq Z_{n}^{\Phi_{f}}(t) \cdot \sum_{s=0}^{n} \sum_{P \in \mathcal{P}_{s}} \delta^{n-s} K^{2 t(n-s)}=Z_{n}^{\Phi_{f}}(t) \cdot \sum_{s=0}^{n}\left(\begin{array}{l}
n \\
s
\end{array}\right)\left(\delta K^{2 t}\right)^{n-s} \\
& =\left(1+\delta K^{2 t}\right)^{n} \cdot Z_{n}^{\Phi_{f}}(t) \leq\left(1+\delta K^{2 d}\right)^{n} \cdot Z_{n}^{\Phi_{f}}(t) .
\end{aligned}
$$

So $\underline{P}(t) \leq \underline{P}_{f}(t)+\log \left(1+\delta K^{2 d}\right) \leq \underline{P}_{f}(t)+\delta K^{2 d}$ as claimed.

We can use the preceding result to determine the Hausdorff dimension of $J(\Phi)$ whenever, for every $\delta>0$, the subsystem $\Phi_{f}$ can be chosen such that Bowen's formula holds for $\Phi_{f}$. In particular, the result shows that very small pieces are irrelevent for the 
computation of the pressure function. This means that, given a bound on the number of pieces at each level, we can always restrict to a system satisfying a corresponding balancing condition:

5.2. Corollary (Balance from growth restrictions). Let $\Phi$ be a finite non-autonomous conformal IFS, and let $t_{0}>0$. Also let $\alpha_{n} \geq 1$ be any sequence such that $\alpha_{n} \rightarrow \infty$.

Then there exists a subsystem $\Phi_{f}$, with index sets $I_{f}^{(n)} \subset I^{(n)}$ of $\Phi$ such that $\underline{P}^{\Phi_{f}}(t)=$ $\underline{P}^{\Phi}(t)$ for all $t \geq t_{0}$, and such that

$$
\rho_{n}\left(\Phi_{f}\right) \leq \alpha_{n} \cdot\left(\# I^{(n)}\right)^{\frac{1}{t_{0}}}
$$

for all $n$. Here

$$
\rho_{n}\left(\Phi_{f}\right):=\max _{a, b \in I_{f}^{(n)}} \frac{\left\|D \varphi_{a}^{(n)}\right\|}{\left\|D \varphi_{b}^{(n)}\right\|} .
$$

Proof. Let us set

$$
\varepsilon_{n}:=\bar{c}_{n} \cdot \alpha_{n}^{-1} \cdot\left(\# I^{(n)}\right)^{-\frac{1}{t_{0}}}
$$

and let $I_{f}^{(n)}$ consist of all indices $a \in I^{(n)}$ for which $\left\|D \varphi_{a}^{(n)}\right\| \geq \varepsilon_{n}$. Then $\rho_{n}\left(\Phi_{f}\right)$ has the required property.

Furthermore, for $t \geq t_{0}$,

$$
\begin{aligned}
\sum_{j \in I^{(n)} \backslash I_{f}^{(n)}}\left\|D \varphi_{j}^{(n)}\right\|^{t} & \leq \# I^{(n)} \cdot \varepsilon_{n}^{t} \\
& =\bar{c}_{n}^{t} \cdot\left(\# I^{(n)}\right)^{1-\frac{t}{t_{0}}} \cdot \alpha_{n}^{-t} \leq \alpha_{n}^{-t} \cdot \sum_{j \in I_{f}^{(n)}}\left\|D \varphi_{j}^{(n)}\right\|^{t} .
\end{aligned}
$$

Since $\alpha_{n}$ tends to zero, it follows from Proposition 5.1 that $\underline{P}^{\Phi_{f}}(t)=\underline{P}^{\Phi}(t)$, as claimed.

This allows us to complete the proof of the positive part of Theorem 1.1.

5.3. Theorem (Bowen's formula for systems of sub-exponential growth). Suppose that $\Phi$ is a conformal iterated function system that is subexponentially bounded, i.e.

$$
\lim _{n \rightarrow \infty} \frac{1}{n} \log \# I^{(n)}=0 .
$$

Then Bowen's Formula holds for $\Phi$, i.e.

$$
\operatorname{HD}(J(\Phi))=B(\Phi) .
$$

Proof. Recall that always $\operatorname{HD}(J(\Phi)) \leq B(\Phi)$ by Lemma 2.8. If $B(\Phi)=0$, there is nothing to prove, so let us assume that $B(\Phi)>0$, and fix some $t_{0} \in(0, B(\Phi))$.

By Corollary [5.2, there is a subsystem $\Phi_{f}$ of $\Phi$ such that $\underline{P}^{\Phi_{f}}(t)=\underline{P}^{\Phi}(t)$ for all $t \geq t_{0}$, and such that

$$
\rho_{n}\left(\Phi_{f}\right) \leq n \cdot\left(\# I^{(n)}\right)^{\frac{1}{t_{0}}}
$$

In particular, $B\left(\Phi_{f}\right)=B(\Phi)$ and $\Phi_{f}$ is weakly balanced. By Corollary 4.3 , we see that

$$
B(\Phi)=B\left(\Phi_{f}\right)=\operatorname{HD}\left(J\left(\Phi_{f}\right)\right) \leq \operatorname{HD}(J(\Phi)) \leq B(\Phi) .
$$

Likewise, we can complete the positive part of Theorem 1.2 . 
5.4. Theorem. Suppose that $\Phi$ is a non-autonomous conformal iterated function system such that

$$
\limsup _{n \rightarrow \infty} \frac{1}{n} \log \# I^{(n)}<\infty
$$

If $\bar{c}_{n} \rightarrow 0$ as $n \rightarrow \infty$, if $\mathrm{HD}(J(\Phi))=0$, or if $B(\Phi)=2$, then Bowen's formula holds.

Proof. As in the proof of the preceding theorem, there is a finite subsystem $\Phi_{f}$ where $\rho_{n}\left(\Phi_{f}\right)$ grows at most exponentially, and for which $B\left(\Phi_{f}\right)=\Phi_{f}$. The result follows from Corollary 4.4 resp. Theorem 4.6.

\section{Counterexamples to Bowen's Formula}

We now turn to completing the proof of Theorem 1.1 by giving counterexamples that show that Theorem 5.3 is best possible.

6.1. Theorem (Counterexamples to Bowen's formula). Let $0<t_{1}<t_{2}<d$, and let $\varepsilon>0$. Then there exists a perfectly balanced NCIFS $\Phi$ with $\limsup _{j \rightarrow \infty} \frac{1}{j} \log \# I^{(j)} \leq \varepsilon$ such that

$$
\mathrm{HD}(J(\Phi))=t_{1} \text { and } B(\Phi)=t_{2} .
$$

Proof. The idea of the proof is very simple. We begin with a uniformly finite and balanced system $\Psi$ for which $\underline{P}^{\Psi}\left(t_{2}\right)=\bar{P}^{\Psi}\left(t_{2}\right)=0$, hence $\operatorname{HD}(J(\Psi))=t_{2}$, and $\underline{P}\left(t_{1}\right)=$ $\bar{P}\left(t_{1}\right)<\infty$. We then modify the system $\Psi$ at a sequence $\left(n_{k}\right)_{1}^{\infty}$ of times, where $n_{k}$ will grow sufficiently quickly, in such a way that the modified system $\Phi$ still has $B(\Phi)=t_{2}$, but such that the corresponding sets $X_{n_{k}}^{\Phi}$ admit much more efficient covers in terms of $t_{1}$-dimensional Hausdorff measure.

We now provide the details, assuming for simplicity that $d=1$; the general case is completely analogous. Let $X=[0,1]$. The construction begins by choosing a suitable perfectly balanced system $\Psi$ that is periodic, i.e.

$$
\Psi^{(j+m)}=\Psi^{(j)}
$$

for all $j \in \mathbb{N}$ and some $m \in \mathbb{N}$. In other words, up to a renormalization of time, $\Psi$ is a classical iterated function system. This system is going to be chosen such that $\underline{P}^{\Psi}\left(t_{2}\right)=\bar{P}^{\Psi}\left(t_{2}\right)=0$. In order to achieve arbitrarily slow exponential growth, $\Psi$ will be chosen so that furthermore the number of words of length $n$ has small exponential growth in $n$, which can be achieved by artificially inserting long stages where a single contraction by a factor close to one is applied at each step in time. To be explicit, let us define such a system $\Psi$ by

$$
\Psi^{\ell}:= \begin{cases}\{x \mapsto x / 2 ; x \mapsto(x+1) / 2\} & \text { if } \ell \bmod m=0 \\ \{x \mapsto \lambda x\} & \text { otherwise. }\end{cases}
$$

Here the integer $m \geq 1$ will be chosen sufficiently large, depending on $t_{1}$ and $t_{2}$ as indicated below, and

$$
\lambda:=2^{-\frac{1-t_{2}}{t_{2}(m-1)}}
$$

Then, for all $n, k \geq 1$ and all $t \geq 0$, we have

$$
Z_{n}^{\Psi}(t) \leq Z_{n}^{\Psi}(0)=2^{\left\lfloor\frac{n}{m}\right\rfloor} \leq 2^{\frac{n}{m}}
$$


and

$$
Z_{k \cdot m}^{\Psi}\left(t_{2}\right)=\left(2 \cdot\left(\frac{\lambda^{m-1}}{2}\right)^{t_{2}}\right)^{k}=\left(\lambda^{t_{2} \cdot(m-1)} \cdot 2^{1-t_{2}}\right)^{k}=\left(2^{t_{2}-1} \cdot 2^{1-t_{2}}\right)^{k}=1
$$

Claim 1. We have $\underline{P}^{\Psi}\left(t_{2}\right)=\bar{P}^{\Psi}\left(t_{2}\right)=0$. In particular, $B(\Psi)=t_{2}$, and $\mathbb{Z}_{n}^{\Psi}\left(t_{1}\right)$ grows exponentially.

Proof. It follows from (6.2) and the very definition of $\Psi$ that

$$
\lambda^{t_{2} \cdot(m-1)} \leq Z_{n}^{\Psi}\left(t_{2}\right) \leq 1
$$

for all $n \in \mathbb{N}$. Hence

$$
\lim _{n \rightarrow \infty} \frac{1}{n} \log Z_{n}^{\Psi}\left(t_{2}\right)=0
$$

as claimed.

For all $k \geq 1$, we now inductively define positive integers $n_{k}$ and $M_{k}$, and a real number $\lambda_{k} \in(0,1)$. These sequences give rise a non-autonomous iterated function systems $\Phi$ as follows. If $j \neq n_{k}$ for all $k$, we set

$$
\Phi^{(j)}:=\Psi^{(j)},
$$

while

$$
\Phi^{\left(n_{k}\right)}:=\left\{x \mapsto \lambda_{k} x / M_{k}, x \mapsto \lambda_{k}(x+1) / M_{k}, \ldots, x \mapsto \lambda_{k}\left(x+M_{k}-1\right) / M_{k}\right\} .
$$

The system $\Phi$ is perfectly balanced; let us use $c_{n}$ to denote the contraction factor $\underline{c}_{n}=\bar{c}_{n}$ at stage $n$. Note that $c_{n}=\lambda_{k} / M_{k}$ when $n=n_{k}$ and that $c_{n} \in\{\lambda, 1 / 2\}$ for all other values of $n$.

We now specify the inductive construction. The initial value $n_{1}>m$ is arbitrary. Let $k \geq 1$ and suppose that $n_{j}$ has been defined for $1 \leq j \leq k$, while $\lambda_{j}$ and $M_{j}$ have been defined for $1 \leq j<k$. Note that this determines $Z_{n_{k}-1}^{\Phi}(t)$ for all $t$. Then we define

$$
\lambda_{k}:=\frac{1}{\left(Z_{n_{k}-1}^{\Phi}\left(t_{1}\right)\right)^{1 / t_{1}}} \quad \text { and } \quad M_{k}:=\left\lfloor\lambda_{k}^{-\frac{t_{2}}{1-t_{2}}}\right\rfloor
$$

Finally, we choose $n_{k+1}>n_{k}$ sufficiently large that

$$
\log Z_{n_{k+1}-1}^{\Psi}\left(t_{1}\right) / 2<\log Z_{n_{k+1}-1}^{\Phi}\left(t_{1}\right)<2 \log Z_{n_{k+1}-1}^{\Psi}\left(t_{1}\right) .
$$

In particular, $\lambda_{k} \rightarrow 0$ and $M_{k} \rightarrow \infty$. We may also assume that the sequence $n_{k}$ is chosen so that $n_{k} / k \rightarrow \infty$.

Claim 2. We have $\underline{P}^{\Phi}\left(t_{2}\right)=\bar{P}^{\Phi}\left(t_{2}\right)=0$ and $\operatorname{HD}(J(\Phi))=t_{1}$.

Proof. We first turn to considering the sum $Z_{n}^{\Phi}\left(t_{2}\right)$. Since the system is linear, this sum is just the product

$$
\prod_{j=1}^{n} c_{j}^{t_{2}} \# \Phi^{(j)}
$$


When $j \neq n_{k}$ for any $k$, the $j$ th factor above is the same as the corresponding contribution in $Z_{n}^{\Psi}\left(t_{2}\right)$. For $j=n_{k}$,

$$
c_{n_{k}}^{t_{2}} \cdot \# \Phi^{\left(n_{k}\right)}=\frac{\lambda_{k}^{t_{2}}}{M_{k}^{t_{2}}} \cdot M_{k}=\lambda_{k}^{t_{2}} \cdot\left\lfloor\lambda_{k}^{-\frac{t_{2}}{1-t_{2}}}\right\rfloor^{1-t_{2}} \in\left[1-\lambda_{k}, 1\right] .
$$

It follows that

$$
Z_{n}^{\Psi}\left(t_{2}\right) / C^{k} \leq Z_{n}^{\Phi}\left(t_{2}\right) \leq C^{k} \cdot Z_{n}^{\Psi}\left(t_{2}\right)
$$

for a suitable constant $C>1$, where $k=k(n)$ is the largest integer with $n_{k} \leq n$. So

$$
\frac{1}{n}\left|\log Z_{n}^{\Psi}\left(t_{2}\right)-\log Z_{n}^{\Phi}\left(t_{2}\right)\right| \leq \log C \cdot \frac{k(n)}{n} \rightarrow 0,
$$

and hence $\underline{P}^{\Phi}\left(t_{2}\right)=\bar{P}^{\Phi}\left(t_{2}\right)=0$ by Claim 1 .

To show that $\operatorname{HD}(J(\Phi)) \leq t_{1}$, note that $\mathcal{U}_{n_{k}}:=\left\{\left[0, \lambda_{k}\right]\right\}$ covers the images of all maps in $\Phi^{\left(n_{k}\right)}$. Furthermore, we have

$$
Z_{n_{k}-1}^{\Phi}\left(t_{1}\right) \cdot \lambda_{k}^{t_{1}}=1
$$

by definition. Hence Lemma 2.9 implies that $\operatorname{HD}(J(\Phi)) \leq t_{1}$.

Finally, we consider the modified sums $\tilde{Z}_{n}\left(t_{1}\right)$ as defined in (3.2). If $n \neq n_{k}$, then $\tilde{Z}_{n}\left(t_{1}\right)$ agrees with $Z_{n-1}\left(t_{1}\right)$ up to a bounded factor, and $Z_{n-1}\left(t_{1}\right) \rightarrow \infty$ as $n \rightarrow \infty$. (Recall that $B(\Phi)=t_{2}>t_{1}$.) For $n=n_{k}$, we have

$$
\tilde{Z}_{n_{k}}^{\Phi}\left(t_{1}\right)=Z_{n_{k}-1}^{\Phi}\left(t_{1}\right) \cdot M_{k}^{t_{1}} \cdot c_{n_{k}}^{t_{1}}=Z_{n_{k}-1}^{\Phi}\left(t_{1}\right) \cdot \lambda_{k}^{t_{1}}=1 .
$$

Hence $\tilde{P}\left(t_{1}\right)=\liminf \log \tilde{Z}_{n}\left(t_{1}\right) / n \geq 0$, and $\operatorname{HD}(J(\Phi)) \geq t_{1}$ by Lemma 4.2 .

It remains only to estimate the growth of the index sets, which means that we should estimate the growth of $M_{k}$ compared to $n_{k}$. We have

$$
\begin{aligned}
\log M_{k} & \leq \frac{t_{2}}{1-t_{2}}\left|\log \lambda_{k}\right|=\frac{t_{2}}{t_{1}\left(1-t_{2}\right)}\left|\log Z_{n_{k}-1}^{\Phi}\left(t_{1}\right)\right| \\
& <\log 2 \frac{t_{2}}{t_{1}\left(1-t_{2}\right)}\left|\log Z_{n_{k}-1}^{\Psi}\left(t_{1}\right)\right| \\
& \leq 2 \log 2 \frac{t_{2}}{t_{1}\left(1-t_{2}\right)} \frac{n_{k}}{m} \leq 4 \cdot \frac{t_{2}}{m t_{1}\left(1-t_{2}\right)} n_{k} .
\end{aligned}
$$

If $m$ was chosen sufficiently large, we thus have $\log M_{k} \leq \varepsilon \cdot n_{k}$, as desired.

Remark. The set $J(\Phi)$ in the above proof is a "partial homogeneous Moran set", in the sense defined in [19], and as such it is well-known how to calculate its Hausdorff dimension; see e.g. [19, Theorem C]. We decided to include a self-contained proof for the reader's convenience, particularly since the notation used in the above reference is different from ours.

With minor modification, the same construction can also be used to show that our results on systems with at most exponential growth cannot be extended to any larger growth conditions, completing the proof of Theorem 1.2.

6.2. Theorem (Counterexamples of superexponential growth). Let $\left(\alpha_{n}\right)_{n \in \mathbb{N}}$ be any sequence of positive integers such that $\log \alpha_{n} / n \rightarrow \infty$. Then there exists a perfectly balanced NCIFS $\Phi$ such that: 
(a) $\# I^{(n)} \leq \alpha_{n}$ for all $n$;

(b) $\bar{c}_{n} \rightarrow 0$ as $n \rightarrow \infty$;

(c) $\operatorname{HD}(J(\Phi))=0$ and $B(\Phi)=d$.

Proof. For $\varepsilon \in(0, d / 2)$, set $t_{1}=t_{1}(\varepsilon):=\varepsilon$ and $t_{2}:=t_{2}(\varepsilon):=d-\varepsilon$. Also let $\Psi=\Psi(\varepsilon)$ be a perfectly balanced autonomous linear system with $B(\Psi(\varepsilon))=t_{2}(\varepsilon)$ such that the contraction factor of the linear maps in $\Psi$ tends to zero as $\varepsilon \rightarrow 0$. For example, in dimension one we can take

$$
\Psi=\{x \mapsto \rho x, x \mapsto \rho(x+1), x \mapsto \rho(x+L-1)\}, \quad \rho=L^{-\frac{1}{t_{2}}}, \quad L=L(\varepsilon) \rightarrow \infty .
$$

We can apply the construction from the previous proof to $t_{1}, t_{2}$ and $\Psi$. The result is a nonautonomous system $\Theta(\varepsilon)$ whose Bowen dimension is $t_{2}$, whose limit set has Hausdorff dimension $t_{1}$, and such that the growth of $\Theta(\varepsilon)$ is at most exponential. (According to (6.3), the exponent of growth tends to infinity at most like $\log L / \varepsilon^{2}$.)

Now let $\varepsilon_{k} \rightarrow 0$. The desired system $\Phi$ is obtained by letting $\Phi^{(j)}$ agree with $\Theta\left(\varepsilon_{k}\right)^{(j)}$ for $N_{k-1} \leq j<N_{k}$, where $N_{k}$ is an increasing sequence of natural numbers. Since $Z_{n}^{\Theta\left(\varepsilon_{k}\right)}(t) \rightarrow \infty$ for all $t<t_{2}\left(\varepsilon_{k}\right)$, we can ensure that $Z_{n}^{\Phi}(t) \rightarrow \infty$ for all $t<d$, and hence $B(\Phi)=d$. Similarly, using the same covers as in the proof of Theorem 6.1, we ensure that $\operatorname{HD}\left(J(\Phi)=0\right.$. By choice of $\Psi$, and by construction, $\bar{c}_{n} \rightarrow 0$ as $n \rightarrow \infty$.

Finally (again, assuming $N_{k}$ is chosen to grow sufficiently quickly), we can ensure that $\# I^{(n)} \leq \alpha_{n}$ for all sufficiently large $n$ (since each $\Theta\left(\varepsilon_{k}\right)$ has some finite exponential growth rate, but $\alpha_{n}$ tends to infinity superexponentially). By modifying $\Phi$ at finitely many initial stages, we can ensure that the condition is satisfied for all $n$, as claimed.

To conclude this section, we shall discuss a very natural system (of super-exponential growth) where Bowen's formula fails.

This example arise from the study of continued fraction expansions of real numbers. These expansions can be studied by considering the system of conformal maps

$$
\varphi_{n}: x \mapsto 1 /(n+x) \quad(n \geq 1)
$$

In order to fit into our framework, where we require uniform contraction, we should only allow $n \geq 2$, As we will study numbers whose continued fraction expansions tend to $\infty$, this is not a serious restriction.

Consider, for some $K \geq 2$ and $\alpha>1$, the index sets

$$
I^{(n)}:=\left\{j \in \mathbb{N}: K^{\alpha^{n}} \leq j<K^{\alpha^{n+1}}\right\} .
$$

The limit set of the associated nonautonomous IFS then consists of those numbers $x \in$ $(0,1)$ for which the continued fraction expansion $\left(a_{n}\right)_{n \in \mathbb{N}}$ satisfies $K^{\alpha^{n}} \leq a_{n} \leq K^{\alpha^{n+1}}$.

6.3. Proposition. For every $\alpha>1$ and $K \geq 2$, the nonautonomous conformal iterated function system $\Psi$ defined by (6.5) and (6.4) satisfies

$$
\operatorname{HD}(J(\Psi))=\frac{1}{1+\alpha}<B(\Psi)=\frac{1}{2} .
$$

Sketch of proof. (In the following, we shall only indicate the order of magnitude of the quantities that occur. Turning this sketch into a proof with rigorous estimates is a straightforward, but not very enlightening, exercise.) 
We begin by calculating the Bowen dimension. Since $\left\|\varphi_{j}^{\prime}\right\|=1 / j^{2}$, this means that we should estimate the sum

$$
\sum_{j=j_{1}}^{j_{2}}\left\|\varphi_{j}^{\prime}\right\|^{t}=\sum_{j=j_{1}}^{j_{2}} j^{-2 t}
$$

where $j_{1}=\left\lceil K^{\alpha^{n}}\right\rceil$ and $j_{2}=\left\lceil K^{\alpha^{n+1}}\right\rceil-1$. For large $n$, the value of this sum can be approximated (up to an error that is negligible for our purposes) by the integral

$$
\int_{j_{1}}^{j_{2}} x^{-2 t} d x=\frac{1}{1-2 t}\left(j_{2}^{1-2 t}-j_{1}^{1-2 t}\right)=\frac{j_{2}^{1-2 t}}{1-2 t}\left(1-\left(\frac{j_{1}}{j_{2}}\right)^{1-2 t}\right) .
$$

So the $j$-th summand in the sum $Z_{n}(t)$ is on the order of magnitude of $K^{\alpha^{j+1}(1-2 t)}$. Summing the geometric series in the exponent, $Z_{n}(t)$ itself behaves like

$$
K^{(1-2 t) \cdot \frac{\alpha^{n+2}}{\alpha-1}} \text {. }
$$

It follows that the pressure is positive infinite for $t<1 / 2$ and negative infinite for $t>1 / 2$. Hence the Bowen dimension $B(\Psi)$ is equal to $1 / 2$, as claimed.

Luczak [8] showed that the set of points whose continued fraction expansion satisfies $a_{n} \geq K^{\alpha^{n}}$ has Hausdorff dimension $1 /(1+\alpha)$, so $\operatorname{HD}(J(\Psi)) \leq 1 /(1+\alpha)$.

Thus it remains to prove that $\operatorname{HD}(J(\Psi)) \geq 1 /(1+\alpha)$. We shall use Theorem 3.2 . First observe that

$$
\# I^{(n)} \cdot \underline{c}_{n} \geq \mathrm{const} \cdot K^{-\alpha^{n+1}}
$$

which means that

$$
\log \tilde{Z}_{n}(t)=\log Z_{n-1}(t)+t \log \left(\# I^{(n)} \cdot \underline{c}\right)
$$

grows like

$$
\begin{aligned}
\left(\frac{(1-2 t) \cdot \alpha^{n+1}}{\alpha-1}-t \alpha^{n+1}\right) \cdot \log K & =\frac{\alpha^{n+1} \log K}{\alpha-1} \cdot(1-2 t-t \alpha+t) \\
& =\frac{\alpha^{n+1} \log K}{\alpha-1} \cdot(1-t(1+\alpha))
\end{aligned}
$$

For $t<1 /(1+\alpha)$, this quantity tends to $+\infty$ exponentially fast, so that $\tilde{Z}_{n}(t)$ grows superexponentially. On the other hand,

$$
\rho_{n} \leq K^{2 \alpha^{n+1}}
$$

and hence the quantity

$$
1+\log \max _{j \leq n} \rho_{j}
$$

grows only exponentially with $n$. Hence $\operatorname{HD}(J(\Psi)) \geq 1 /(1+\alpha)$ by Theorem 3.2 .

\section{Hausdorff and Packing Measures}

In this short section, we consider balanced and uniformly finite systems, where we can fully charcterize the cases when the $h$-dimensional Hausdorff measure $\mathrm{H}_{h}(J(\Phi))$ is finite, positive or infinite, where $h=B(\Phi)=\operatorname{HD}(J(\Phi))$. The answer depends on the value of $\liminf _{n \rightarrow \infty} Z_{n}(h)$. 
7.1. Theorem. Let $\Phi$ be a balanced and uniformly finite non-autonomous conformal iterated function system, and let $h=B(\Phi)$.

Then the h-dimensional Hausdorff measure $\mathrm{H}_{h}(J(\Phi))$ is infinite, finite or equal to zero according to whether

$$
\liminf _{n \rightarrow \infty} Z_{n}(h)
$$

is infinite, finite or equal to zero, respectively.

Proof. The proof of Lemma 2.9 produces a constant $C>0$ such that that

$$
\mathrm{H}_{h}(J(\Phi)) \leq C \liminf _{n \rightarrow \infty} Z_{n}(h) .
$$

Hence we only need to show that $\mathrm{H}_{h}(J(\Phi))$ is positive when the lim inf above is positive, and infinite when the liminf is infinite. To do so, we note that the proof of Theorem 3.2 actually gives that

$$
m(B) \leq \text { const } \cdot r^{h}
$$

for any ball of radius $r$ when the assumptions of that theorem are satisfied, i.e. when

$$
\liminf _{n \rightarrow \infty} \frac{\tilde{Z}_{n}(h)}{1+\log \max _{j \leq n} \rho_{n}}
$$

is positive. Furthermore, if this lim inf is infinite, then the proof shows even that

$$
\frac{m(B)}{r^{h}} \rightarrow 0
$$

as $r \rightarrow 0$. By the Converse Frostman Lemma, this means that $\mathrm{H}_{h}(J(\Phi))$ is positive resp. infinite.

By assumption, our system is balanced, so $\rho_{n}$ is uniformly bounded. Furthermore, the system is uniformly finite, and hence $\tilde{Z}_{n}(h)$ differs from $Z_{n}(h)$ by at most a multiplicative constant. This means that the liminf in (7.1) is comparable to $\lim _{n \rightarrow \infty} Z_{n}(h)$, and the theorem is proved.

\section{Systems with a Countably Infinite Alphabet}

We now turn to the study of non-autonomous systems with infinite countable alphabets. For simplicity, we shall assume that all stages are infinite, and share the same alphabet. That is, $I^{(n)}=\mathbb{N}$ for all $n \in \mathbb{N}$. The reader may find it helpful to imagine the pieces at level $n$ being labelled in decreasing order of size (i.e., $\left(\left\|D \varphi_{j}^{(n)}\right\|\right)_{j \in \mathbb{N}}$ is a decreasing sequence), although we will not formally require this.

One of the technical differences that arise in the infinite case is that some of the sums $Z_{n}(t)$ may be infinite (for finite $n$ ), This will cause the sums $Z_{n^{\prime}}(t)$ to be infinite for all $n^{\prime} \geq n$, and hence makes them rather unsuitable for predicting the geometry of the limit set at small scales without prior restrictions. Clearly this happens if and only if one of the individual sums

$$
Z_{1}^{(m)}(t)=\sum_{j \in I^{(m)}}\left\|D \varphi_{j}^{(m)}\right\|^{t}
$$

is infinite for $m \leq n$. In practical applications, for fixed $t$ these sums will usually be either finite for all $m$ or infinite for all $m$, and in the following we shall consider only systems where this is the case. As soon as we deal with this technical issue, Corollary 8.3 and 
with Corollary 8.6 provide us immediately with a large class of systems where Bowen's formula holds.

8.1. Definition. We say that an infinite non-autonomus conformal iterated function system $\Phi=\left\{\Phi^{(n)}\right\}_{n=1}^{\infty}$ belongs to class $\mathcal{M}$ if the following conditions are satisfied for all $t \in(0, d)$ and all $\varepsilon>0$.

(a) The sums $Z_{1}^{(n)}(t)$ are either infinite for all $n$ or finite for all $n$.

(b) If $Z_{1}(t)<\infty$, then

$$
\lim _{n \rightarrow \infty} \frac{\sum_{k \leq e^{\varepsilon n}}\left\|D \varphi_{k}^{(n)}\right\|^{t}}{Z_{1}^{(n)}(t)}=1
$$

(c) If $Z_{1}(t)=\infty$, then

$$
\lim _{n \rightarrow \infty} \sum_{k \leq e^{\varepsilon n}}\left\|D \varphi_{k}^{(n)}\right\|^{t}=\infty .
$$

Of course the key point of this technical definition is that, given $\Phi \in \mathcal{M}$, we can restrict to a well-controlled finite subsystem.

8.2. Observation. If $\Phi \in \mathcal{M}$, then there is a finite and subexponentially bounded subsystem $\Phi_{F}$, determined by index sets $I_{F}^{(n)} \subset \mathbb{N}$, such that $\underline{P}^{\Phi_{F}}(t)=\underline{P}^{\Phi}(t)$ for all $t>0$.

Proof. First observe that, for each $n$, we may reorder the sequence $\left(\varphi_{k}^{(n)}\right)_{k \in \mathbb{N}}$ without affecting the limit set of $\Phi$, the pressure function, or the value $Z_{1}^{(n)}(t)$ (for all $t$ ). Among all possible such reorderings, the sum $\sum_{k \leq e^{\varepsilon n}}\left\|D \varphi_{k}\right\|^{t}$ will be minimized if the sequence $\left(\left\|D \varphi_{k}^{(n)}\right\|\right)_{k \in \mathbb{N}}$ is nonincreasing. Hence we may assume in the following that each $\Phi^{(n)}$ is ordered in this manner.

Let $T$ denote the interval of positive values of $t$ for which $Z_{1}(t)<\infty$. Let $t \in T$. Then, by letting $\varepsilon$ tend to zero in (b) and diagonalizing, there is a sequence $\left(\kappa_{n}\right)_{n \in \mathbb{N}}$ of positive integers such that $\kappa_{n}=\kappa_{n}(t)$ grows at most subexponentially in $n$ and such that

$$
\lim _{n \rightarrow \infty} \frac{\sum_{k>\kappa_{n}(t)}\left\|D \varphi_{k}^{(n)}\right\|^{\tau}}{\sum_{k \leq \kappa_{n}(t)}\left\|D \varphi_{k}^{(n)}\right\|^{\tau}}=0
$$

for $\tau=t$. Since the sequence $\left\|D \varphi_{k}^{(n)}\right\|$ is nonincreasing in $k$, we automatically obtain (8.1) also for all $\tau>t$. Indeed, we can write

$$
\frac{\sum_{k>\kappa_{n}(t)}\left\|D \varphi_{k}^{(n)}\right\|^{\tau}}{\sum_{k \leq \kappa_{n}(t)}\left\|D \varphi_{k}^{(n)}\right\|^{\tau}}=\frac{\sum_{k>\kappa_{n}(t)}\left(\frac{\left\|D \varphi_{k}^{(n)}\right\|}{\left\|D \varphi_{\kappa_{n}(t)}\right\|}\right)^{\tau}}{\sum_{k \leq \kappa_{n}(t)}\left(\frac{\left\|D \varphi_{k}^{(n)}\right\|}{\left\|D \varphi_{\kappa_{n}(t)}\right\|}\right)^{\tau}} .
$$

and the enumerator of the expression on the right-hand side is decreasing in $\tau$, while the denominator is increasing.

Letting $t$ tend towards the lower endpoint of $T$ and applying another diagonalization, we can choose a sequence $\kappa_{n}$, independently of $t$, such that (8.1) holds for all $\tau \in T$. 
Likewise, we can choose a sequence $K_{n}$ such that

$$
\lim _{n \rightarrow \infty} \sum_{k \leq K_{n}}\left\|D \varphi_{k}^{(n)}\right\|^{t}=\infty
$$

for all $t \in(0, d) \backslash T$, and such that $K_{n}$ grows at most subexponentially.

Now set $I_{F}^{(n)}:=\left\{k \in \mathbb{N}: k \leq \max \left(\kappa_{n}, K_{n}\right)\right\}$. Then $\underline{P}^{\Phi_{F}}(t)=\underline{P}^{(\Phi)}(t)$ for all $t \in T$ by Proposition 5.1. On the other hand, we have $\underline{P}^{\Phi_{f}}(t)=\infty=\underline{P}^{\Phi}(t)$ for all $t \notin T$.

\subsection{Corollary. Bowen's formula holds for all $\Phi \in \mathcal{M}$.}

Proof. By Theorem 1.1, Bowen's formula holds for the subsystem constructed in the previous observation. So

$$
\operatorname{HD}\left(J\left(\Phi_{F}\right)\right) \leq \operatorname{HD}(J(\Phi)) \leq B(\Phi)=B\left(\Phi_{F}\right)=\operatorname{HD}\left(J\left(\Phi_{F}\right)\right) .
$$

The requirements of Definition 8.1 are technical and look somewhat awkward. Hence we shall now define a subclass of $\mathcal{M}$ whose definition is easy to define and verify. (In particular, this class contains all infinite autonomous conformal iterated function systems.) This class will be used in Section 9 to establish Bowen's formula for random conformal iterated function systems.

\subsection{Definition.}

We say that an infinite non-autonomous system $\Phi$ is evenly varying if there is a sequence $\left(\gamma_{i}\right)_{i \in \mathbb{N}}$ of positive real numbers and a constant $c \geq 1$ such that

$$
\frac{\gamma_{i}}{c} \leq\left\|D \varphi_{i}^{(n)}\right\| \leq c \cdot \gamma_{i}
$$

The class of all evenly varying systems is denoted EV.

\subsection{Proposition. $\mathrm{EV} \subset \mathcal{M}$.}

Proof. Let $\varphi \in \mathrm{EV}$, and let $\left(\gamma_{i}\right)$ and $c$ be as above. By definition, $Z_{1}^{(n)}(t)$ is proportional to $\sum_{i} \gamma_{i}^{t}$ for all $n$ and $t$. Hence, for fixed $t$, these sums are either finite for all $n$ or infinite for all $n$, depending on whether $\left(\gamma_{i}^{t}\right)$ is summable.

Suppose that $\sum_{i} \gamma_{i}^{t}<\infty$, and let $m, n \in \mathbb{N}$. Then

$$
\frac{\sum_{i=1}^{m}\left\|D \varphi_{i}^{(n)}\right\|^{t}}{Z_{1}^{(n)}(t)} \geq 1-\frac{c^{2}}{\gamma_{1}^{t}} \cdot \sum_{i=m+1}^{\infty} \gamma_{i}^{t} .
$$

So this quotient tends to 1 uniformly in $n$ as $m \rightarrow \infty$, which proves condition (b) of Definition 8.1.

If $\sum_{i} \gamma_{i}^{t}=\infty$, then likewise

$$
\sum_{i=1}^{m}\left\|D \varphi_{i}^{(n)}\right\|^{t} \geq \frac{1}{c} \sum_{i=1}^{m} \varphi_{i}^{t}
$$

tends to infinity uniformly in $n$ as $\mathrm{m} \rightarrow \infty$, proving condition (c) of Definition 8.1, 
8.6. Corollary. Bowen's formula holds for all $\Phi \in \mathrm{EV}$.

\section{Random Conformal Iterated Function Systems}

In this section, we briefly discuss the connection of our results with the study of random conformal iterated function systems, as developed in [17]. Apart from the main results of the theory, the reader may find there an extended discussion of random systems, a number of examples, and a large literature. Our goal is to reprove, generalize, and strengthen the main results regarding the Hausdorff dimensions of limit sets of these systems.

A random CIFS (on a set $X \subset \mathbb{R}^{d}$ as in Section 2) consists of

- an invertible ergodic transformation $T: \Lambda \rightarrow \Lambda$ preserving a probability measure $m$ on a measure space $\Lambda$;

- an alphabet $I$ (finite or countably infinite);

- for every $\lambda \in \Lambda$, an autonomous conformal iterated function system $\Psi_{\lambda}=\left\{\varphi_{i}^{\lambda}\right.$ : $i \in I\}$ on such that the maps $\lambda \mapsto \varphi_{i}^{\lambda}(x)$ are measurable for every $x \in X$.

Thus for every $\lambda \in \Lambda$, there is an associated non-autonomous system

$$
\Phi_{\lambda}=\left\{\Phi_{\lambda}^{(n)}\right\}_{n=1}^{\infty},
$$

where

$$
\Phi_{\lambda}^{(n)}=\left\{\varphi_{i}^{T^{n}(\lambda)}: i \in I\right\} .
$$

Since $T$ is ergodic and $\underline{P}^{\Phi_{\lambda}(t)}=\underline{P}^{\Phi_{T(\lambda)}(t)}$, we have that $\underline{P}^{\Phi_{\lambda}}(t)$ is constant for almost all $\lambda \in \Lambda$. In [17] this constant was denoted by $\mathcal{E} P(t)$ and was characterized by a formula which justifies its name - the expected pressure.

We are primarily interested in the limit set $J\left(\Phi_{\lambda}\right)$, and particularly its Hausdorff dimension, for almost every $\lambda \in \Lambda$. To state our main result, let us define, for every $i \in I$,

$$
\underline{M}_{i}:=\operatorname{ess} \inf \left\{\left\|D \varphi_{i}^{\lambda}\right\|: \lambda \in \Lambda\right\} \quad \text { and } \quad \bar{M}_{i}:=\operatorname{ess} \sup \left\{\left\|D \varphi_{i}^{\lambda}\right\|: \lambda \in \Lambda\right\} .
$$

9.1. Theorem. Let $\Phi$ be a random conformal iterated function system. If the alphabet $I$ is infinite, assume that

$$
\sup _{i \in I} \frac{\bar{M}_{i}}{\underline{M}_{i}}<\infty
$$

Then

$$
\operatorname{HD}\left(J\left(\Phi_{\lambda}\right)\right)=\inf \{t \geq 0: \mathcal{E} P(t) \leq 0\},
$$

for almost every $\lambda \in \Lambda$, where $\mathcal{E} P(t)$, the expected pressure, is the expected value of $\underline{P}^{\Phi_{\lambda}}(t)$.

Remark 1. For infinite $I$, this reproves [17, Theorem 3.18] in an entirely different way. For finite $I$, [17, Theorem 3.18] requires the assumption that $\underline{M}_{i}>0$ for all $i \in I$, while we do not require any additional assumptions in this case.

Proof. It suffices to prove that, for almost every $\lambda$, $\Phi_{\lambda}$ satisfies Bowen's formula. If $I$ is finite, this directly follows from Theorem 1.1. On the other hand, if $I$ is infinite, then it follows from the following observation. 
Claim. If $I$ is infinite and $\Phi$ satisfies (9.1), then $\Phi_{\lambda} \in \mathrm{EV}$ for almost every $\lambda \in \Lambda$.

Proof. Let $\Delta \geq 1$ be such that $\bar{M}_{i} \leq \Delta \underline{M}_{i}$ for all $i$. Then, for all $i \in I$ and almost every $\lambda \in \Lambda$,

$$
\underline{M}_{i} \leq\left\|D \varphi_{i}^{T^{n}(\lambda)}\right\| \leq \bar{M}_{i} \leq \Delta \underline{M}_{i} .
$$

So, putting $c:=\Delta$ and $\gamma_{i}:=\underline{M}_{i}$ for all $i$, we directly see that $\Phi_{\lambda} \in \mathrm{EV}$.

This concludes the proof of the Theorem.

\section{Continuity of Pressure and Hausdorff Dimension}

In this section, we shall prove some results regarding the continuity of pressure and Hausdorff dimension. Throughout the section, we will fix the sets $X \subset \mathbb{R}^{d}$ and $U \supset X$ involved in the definition of a non-autonomous conformal IFS, as well as the bounded distortion constant $K$ and the contraction constant $\eta$ (together with the notion of when " $m$ is large enough" for uniform contraction in Definition 2.1). All systems considered in this section are assumed to satisfy Definition 2.1 with this choice of constants, so that the classes of systems studied here implicitly depend on these initial choices.

If $\Phi$ and $\Psi$ are two arbitrary autonomous conformal iterated function systems (acting on the same set $X)$ with the same finite alphabet $I$, then $d(\Phi, \Psi)$, the distance between $\Phi$ and $\Psi$, is defined as follows:

$$
d(\Phi, \Psi)=\max \left\{\max \left\{\left\|\varphi_{a}-\psi_{a}\right\|_{\infty},\left\|D \varphi_{a}-D \psi_{a}\right\|_{\infty}\right\}: e \in I\right\} .
$$

Now, let $J=\left(I^{(k)}\right)_{k=1}^{\infty}$ be an arbitrary sequence of finite sets. If $\Phi=\left\{\Phi^{(n)}\right\}_{n=1}^{\infty}$ and $\Psi=\left\{\Psi^{(n)}\right\}_{n=1}^{\infty}$ are arbitrary non-autonomous conformal iterated function systems with the alphabets $J=\left(I^{(k)}\right)_{k=1}^{\infty}$, then $d_{u}(\Phi, \Psi)$, the distance between $\Phi$ and $\Psi$, defined as follows:

$$
d_{u}(\Phi, \Psi)=\sup \left\{d\left(\Phi^{(n)}, \Psi^{(n)}\right): n \geq 1\right\} \leq \max \{2, \operatorname{diam}(X)\} .
$$

The subscript $u$ indicates here that the distance is "uniform" with respect to all $n \in \mathbb{N}$.

By $\mathbb{K}_{u}(X, J)$ we denote the space of all non-autonomous conformal iterated function systems with alphabet $J$, acting on $X$ and topologized by the metric $d_{u}$, for which the following two conditions are satisfied:

(Ka) there is $\kappa>0$ such that $\inf \left\{\left|D \varphi_{a}^{(n)}(x)\right|: n \geq 1, a \in I^{(n)}, x \in X\right\}>\kappa$.

(Kb) The family of functions $\left.\left\{\mid D \varphi_{a}^{(n)}\right) \mid: n \geq 1, a \in I^{(n)}\right\}$ is uniformly continuous; i.e.

$$
\begin{aligned}
\forall(\varepsilon>0) & \exists(\delta>0) \quad \forall(n \geq 1) \quad \forall\left(a \in I^{(n)}\right) \\
& \|x-y\|<\delta \Rightarrow|| D \varphi_{a}^{(n)}(x)|-| D \varphi_{a}^{(n)}(y) \|<\varepsilon .
\end{aligned}
$$

Remark. Note that property (Ka) implies, in particular, that the systems we consider here are uniformly finite. (Infinite systems will be considered at the end of the section.)

Property $(\mathrm{Kb})$ is a strengthening of the uniform distortion property of $\Phi$. Observe that this property is, again, automatic in dimensions $\geq 2$.

10.1. Observation. Observe that if all finite sets $I^{(k)}, k \geq 1$, are the same, say equal to $I$, and $\Phi$ is an autonomous system with alphabet $I$ acting on $X$, then $\Phi \in \mathbb{K}_{u}(X, J)$, where $J=(I)_{n=1}^{\infty}$. 
We now prove the following auxiliary result, asserting roughly that if the $d$ metric of two systems is small, then the distance between all the corresponding contractions $\varphi_{\omega}$ and $\psi_{\omega}$ for all finite words $\omega$ are also small.

10.2. Lemma. If $\Phi, \Psi \in \mathbb{K}_{u}(X, J)$, then for all positive integers $m \leq n$, all $\omega \in I^{m, n}$, and all $x \in X$, we have

$$
\left|\varphi_{\omega}^{m, n}(x)-\psi_{\omega}^{m, n}(x)\right| \leq(1-\eta)^{-1} d_{u}(\Phi, \Psi)
$$

Proof. Let $\omega_{*}=\omega_{m} \omega_{m+1} \ldots \omega_{n-1}$. We have

$$
\begin{aligned}
\mid \varphi_{\omega}^{m, n}(x) & -\psi_{\omega}^{m, n}(x) \mid \\
& \leq\left|\varphi_{\omega^{*}}^{m, n-1}\left(\varphi_{\omega_{n}}^{(n)}(x)\right)-\varphi_{\omega^{*}}^{m, n-1}\left(\psi_{\omega_{n}}^{(n)}(x)\right)\right|+\left|\varphi_{\omega^{*}}^{m, n-1}\left(\psi_{\omega_{n}}^{(n)}(x)\right)-\psi_{\omega^{*}}^{m, n-1}\left(\psi_{\omega_{n}}^{(n)}(x)\right)\right| \\
& \leq \eta^{n-m} d(\Phi, \Psi)+\left|\varphi_{\omega^{*}}^{m, n-1}\left(x^{\prime}\right)-\psi_{\omega^{*}}^{m, n-1}\left(x^{\prime}\right)\right|,
\end{aligned}
$$

where $x^{\prime}:=\psi_{\tau_{n}}^{(n)}(x)$. So, proceeding inductively we obtain

$$
\left|\varphi_{\omega}^{m, n}(x)-\psi_{\omega}^{m, n}(x)\right| \leq \sum_{i=0}^{n-m} \eta^{i} d(\Phi, \Psi) \leq(1-\eta)^{-1} d(\Phi, \Psi)
$$

We are ready to prove a continuity property of the lower pressure function $\underline{P}(t)$ in the class $\mathbb{K}_{u}(X, J)$.

10.3. Theorem. For every $t \geq 0$, the lower pressure function

$$
\mathbb{K}_{u}(X, J) \rightarrow \mathbb{R} ; \Psi \mapsto \underline{P}^{\Psi}(t)
$$

is continuous.

Proof. Let $\Phi \in \mathbb{K}_{u}(X, J)$. Note that, by definition of the distance $d_{U}$, there is $\kappa>0$ such that, if $\Psi \in \mathbb{K}_{u}(X, J)$ is sufficiently close to $\Phi$, then $\Phi$ satisfies (Ka) with this choice of $\kappa$.

Fix $\varepsilon>0$, and let $\delta>0$ according to condition $(\mathrm{Kb})$ for the system $\Phi$. Take an arbitrary system $\Psi \in \mathbb{K}(X, J)$ such that

$$
d_{u}(\Psi, \Phi)<(1-\eta) \min \{\varepsilon, \delta\}
$$

We may assume that $\delta>0$ is chosen so small that $\Psi$ satisfies (Ka) for $\kappa$ as mentioned above. 
Fix an arbitrary $n \geq 1, x, y \in X$ and $\omega \in I^{(n)}$. Using also (Kb) for $\Phi$ and Lemma 10.2, we then get

$$
\begin{aligned}
\left|\log \frac{\left|D \psi_{\omega}(x)\right|}{\left|D \varphi_{\omega}(x)\right|}\right| & =\left|\sum_{j=1}^{n} \log \right| D \psi_{\omega_{j}}^{(j)}\left(\psi_{\sigma^{j}(\omega)}^{(j+1)}(x)\right)|-\log | D \varphi_{\omega_{j}}^{(j)}\left(\varphi_{\sigma^{j}(\omega)}^{(j+1)}(x)\right)|| \\
\leq & \sum_{j=1}^{n}|\log | D \psi_{\omega_{j}}^{(j)}\left(\psi_{\sigma^{j}(\omega)}^{(j+1)}(x)\right)|-\log | D \varphi_{\omega_{j}}^{(j)}\left(\varphi_{\sigma^{j}(\omega)}^{(j+1)}(x)\right)|| \\
= & \left.\sum_{j=1}^{n}|\log | D \psi_{\omega_{j}}^{(j)}\left(\psi_{\sigma^{j}(\omega)}^{(j+1)}(x)\right)|-\log | D \varphi_{\omega_{j}}^{(j)}\left(\psi_{\sigma^{j}(\omega)}^{(j+1)}(x)\right) \mid\right)+ \\
& +\left(\log \left|D \varphi_{\omega_{j}}^{(j)}\left(\psi_{\sigma^{j}(\omega)}^{(j+1)}(x)\right)\right|-\log \left|D \varphi_{\omega_{j}}^{(j)}\left(\varphi_{\sigma^{j}(\omega)}^{(j+1)}(x)\right)\right|\right) \mid \\
\leq & \sum_{j=1}^{n}|\log | D \psi_{\omega_{j}}^{(j)}\left(\psi_{\sigma^{j}(\omega)}^{(j+1)}(x)\right)|-\log | D \varphi_{\omega_{j}}^{(j)}\left(\psi_{\sigma^{j}(\omega)}^{(j+1)}(x)\right) \mid+ \\
& +\sum_{j=1}^{n}|\log | D \varphi_{\omega_{j}}^{(j)}\left(\psi_{\sigma^{j}(\omega)}^{(j+1)}(x)\right)|-\log | D \varphi_{\omega_{j}}^{(j)}\left(\varphi_{\sigma^{j}(\omega)}^{(j+1)}(x)\right) \mid \\
\leq & \kappa^{-1} \sum_{j=1}^{n}|| D \psi_{\omega_{j}}^{(j)}\left(\psi_{\sigma^{j}(\omega)}^{(j+1)}(x)\right)|-| D \varphi_{\omega_{j}}^{(j)}\left(\psi_{\sigma^{j}(\omega)}^{(j+1)}(x)\right) \mid+ \\
& \quad+\kappa^{-1} \sum_{j=1}^{n}|| D \varphi_{\omega_{j}}^{(j)}\left(\psi_{\sigma^{j}(\omega)}^{(j+1)}(x)\right)|-| D \varphi_{\omega_{j}}^{(j)}\left(\varphi_{\sigma^{j}(\omega)}^{(j+1)}(x)\right)|| \\
\leq & \kappa^{-1} n d_{u}(\Psi, \Phi)+\kappa^{-1} n \varepsilon \\
\leq & 2 \kappa^{-1} \varepsilon n .
\end{aligned}
$$

Therefore,

$$
K^{-1} \exp \left(-4 \kappa^{-1} \varepsilon n\right) \leq \frac{\left\|D \psi_{\omega}\right\|}{\left\|D \varphi_{\omega}\right\|} \leq K \exp \left(4 \kappa^{-1} \varepsilon n\right) .
$$

Hence,

$$
K^{-t} \exp \left(-4 \kappa^{-1} t \varepsilon n\right) \leq \frac{Z_{n}^{\Psi}(t)}{Z_{n}^{\Psi}(t)} \leq K^{t} \exp \left(4 \kappa^{-1} t \varepsilon n\right)
$$

So,

$$
\left|\underline{\mathrm{P}}_{\Psi}(t)-\underline{\mathrm{P}}_{\Phi}(t)\right|<4 \kappa^{-1} t \varepsilon .
$$

Since $\varepsilon>0$ was arbitary, we are done.

Since the pressure function $[0,+\infty) \ni t \mapsto \underline{P}^{\Psi}(t)$ is strictly decreasing, and, by condition (Ka), our system is uniformly bounded, along with Theorem 1.1, this theorem gives the following.

10.4. Corollary. The Hausdorff dimension function $\mathbb{K}_{u}(X, J) \rightarrow[0, d] ; \Phi \mapsto \operatorname{HD}(J(\Phi))$ is continuous. 
Our consideration in this section up to now applied only to uniformly finite systems. We shall now deal with much more general finite and truly infinite non-autonomous systems.

10.5. Definition. Assume that for every $n \geq 1, I^{(n)}$ is an initial segment of $\mathbb{N}$ (i.e., either $I^{(n)}=\{1, \ldots, m\}$ for some $m$, or $\left.I^{(n)}=\mathbb{N}\right)$. Let $J=\left(I^{(n)}\right)_{j=1}^{\infty}$, and let $\Phi$ be a nonautonomous system with alphabet $J$.

Suppose that $\Phi$ satisfies condition $(K b)$ - i.e., the derivatives $\varphi_{a}^{(n)}$ are uniformly continuous - and that

$$
\inf _{n \in \mathbb{N}}\left\|D \varphi_{a}^{(n)}\right\|>0
$$

for all $a$.

Suppose furthermore that, for every $t \in[0, d]$ and all $\delta>0$, there is $M>0$ such that

(a) the sums $Z_{1}^{(n)}(t)$ are either infinite for all $n$ or finite for all $n$;

(b) if $Z_{1}(t)<\infty$, then, for all $n \geq 1$,

$$
\sum_{k \leq M}\left\|D \varphi_{k}^{(n)}\right\|^{t} \geq(1-\delta) \cdot Z_{1}^{(n)}(t)
$$

(c) if $Z_{1}(t)=\infty$, then, for all $n \geq 1$, then

$$
\sum_{k \leq M}\left\|D \varphi_{k}^{(n)}\right\|^{t} \geq 1 / \delta
$$

Then we say that $\Phi$ belongs to the class $\mathcal{M}_{+}$.

Since $\mathcal{M}_{+} \subset \mathcal{M}$, we immediately obtain the following fact by virtue of Corollary 8.3 , 10.6. Theorem. Bowen's formula holds for all systems $\Phi \in \mathcal{M}_{+}$.

For any systems $\Phi, \Psi \in \mathcal{M}_{+}$define

$$
d_{p}(\Phi, \Psi)=\sum_{n=1}^{\infty} 2^{-n} d\left(\Psi^{(n)}, \Phi^{(n)}\right) .
$$

The number $d_{p}(\Phi, \Psi)$ is obviously a metric on the space $\mathcal{M}$. In contrast to $d_{u}(\Phi, \Psi)$ we refer to it as the pointwise distance between the systems $\Phi$ and $\Psi$.

10.7. Definition. Let $\Phi \in \mathcal{M}_{+}$and $\left(\Phi_{n}\right)_{n=1}^{\infty} \subset \mathcal{M}_{+}$. We say that the sequence $\left(\Phi_{n}\right)_{1}^{\infty}$ $\omega$-converges to $\Phi$ if

(a)

$$
\lim _{n \rightarrow \infty} d_{p}\left(\Phi_{n}, \Phi\right)=0
$$

and

(b) For every $t \in[0, d]$ and all $\delta>0$, the number $M$ in the definition of the class $\mathcal{M}_{+}$can be chosen independently of $n \geq 1$.

Note that in the case of autonomous sequences, $\lambda$-convergence from [14 implies $\omega-$ convergence. Our conditions here are weaker! We shall prove the following. 
10.8. Theorem. If $\Phi \in \mathcal{M}_{+},\left(\Phi_{n}\right)_{1}^{\infty} \subset \mathcal{M}_{+}$and $\left(\Phi_{n}\right)_{1}^{\infty}$ w-converges to $\Phi$, then

$$
\lim _{n \rightarrow \infty} \underline{P}_{\Phi_{n}}(t)=\underline{P}_{\Phi}(t)
$$

for all $t \geq 0$.

Proof. For $M \in \mathbb{N}$, let $\Phi(M)$ and $\Phi_{n}(M)$ denote the finite systems obtained by truncating all alphabets at symbol $M$.

By the definition of class $\mathcal{M}_{+}$and Proposition 5.1, we see that, for every $t$,

$$
\underline{P}^{\Phi(M)}(t) \rightarrow \underline{P}^{\Phi}(t)
$$

as $M \rightarrow \infty$, and similarly for $\Phi_{n}$. Furthermore, the convergence is uniform in $n$.

Furthermore, the system $\Phi(M)$ belongs to the class $\mathbb{K}_{u}(X, \tilde{J})$ (where $\tilde{J}$ is the alphabet of the system $\Phi(M)$ ), and $\Phi_{n}(M)$ converges to $\Phi(M)$ in the $d_{u}$-metric as $n \rightarrow \infty$.

The claim follows.

Recall that, for every non-auonomous system $\Phi$, the pressure function $t \mapsto \mathrm{P}_{\Phi}(t)$ is strictly decreasing throughout its domain of finiteness. Thus we obtain the following immediate consequence of Theorem 10.8 and Theorem 10.6 (Bowen's formula).

10.9. Theorem. If $\Phi \in \mathcal{M}_{+},\left(\Phi_{n}\right)_{1}^{\infty} \subset \mathcal{M}_{+}$and $\left(\Phi_{n}\right)_{1}^{\infty} \omega$-converges to $\Phi$, then

$$
\lim _{n \rightarrow \infty} \operatorname{HD}\left(J_{\Phi_{n}}\right)=\operatorname{HD}(J(\Phi)) .
$$

\section{Appendix: Unbounded orbits in Julia SETS And in Limit Sets of INFINITE AUTONOMOUS IFS}

Let $\Phi=\left\{\varphi_{n}: n \in \mathbb{N}\right\}$ be an infinite (autonomous) conformal iterated function system. (So $I^{(n)}=\mathbb{N}$ for all $n$, and $I^{\infty}=\mathbb{N}^{\mathbb{N}}$.) We are interested in the set of points $x \in J(\Phi)$ that have dense orbits.

More precisely, let $\sigma: \mathbb{N}^{\mathbb{N}} \rightarrow \mathbb{N}^{\mathbb{N}}$ be the shift map, i.e.

$$
\sigma(\omega)_{n}=\omega_{n+1} .
$$

We define

$$
I_{\text {dense }}^{\infty}=\left\{\omega \in \mathbb{N}^{\mathbb{N}}: \overline{\left\{\sigma^{n}(\omega): n \geq 0\right\}}=\mathbb{N}^{\mathbb{N}}\right\},
$$

i.e $I_{\text {dense }}^{\infty}$ consists of all points in $\mathbb{N}^{\mathbb{N}}$ whose forward orbit under the shift map is dense in $\mathbb{N}^{\mathbb{N}}$. Recall the definition of the projection map $\pi_{\Phi}: I^{\infty} \rightarrow J(\Phi)$ from Definition 2.4. We set

$$
J_{\text {dense }}(\Phi)=\pi_{\Phi}\left(I_{\text {dense }}^{\infty}\right) .
$$

Since $J(\Phi)=\pi_{\Phi}\left(I^{\infty}\right)$ and the map $\pi_{\varphi}$ is continuous, we thus get that

$$
\overline{\left\{\pi_{\Phi}\left(\sigma^{n}(\omega)\right): n \geq 0\right\}}=\overline{J(\Phi)} .
$$

11.1. Theorem. If $\Phi$ is an infinite autonomous iterated function system, then

$$
\operatorname{HD}\left(J_{\text {dense }}(\Phi)\right)=\operatorname{HD}(J(\Phi)) .
$$

Remark. This result was previously known in the case when the system $\Phi$ is strongly regular, or at least if it is regular and its entropy is finite (see Corollary 4.4.6 in [10]), but not generally. We note that our proof also works for finite systems, but here the result is well known; see [10, Corollary 4.4.6] 
Proof. Let $\Psi$ be the non-autonomous subsystem of $\Phi$ defined by the index sets

$$
I_{f}^{(n)}:=\{1, \ldots, n\} .
$$

It follows from Proposition 5.1 that $B(\Psi)=B(\Phi)$.

Now let $\left\{\omega^{k}\right\}_{k \in \mathbb{N}}$ be an enumeration of all finite words; i.e., an enumeration of the (countable) set $\bigcup_{j} I^{j}$. Given an arbitrary increasing sequence $\left(n_{k}\right)_{1}^{\infty}$, we define a system $\Theta$ by

$$
\Theta^{(n)}:= \begin{cases}\Psi^{(n)} & \text { if } n \neq n_{k} \text { for all } k \in \mathbb{N} \\ \left\{\varphi_{\omega^{k}}\right\} & \text { if } n=n_{k} \text { for some } k \in \mathbb{N} .\end{cases}
$$

It is clear from this definition that

$$
J(\Theta) \subset J_{\text {dense }}(\Phi) .
$$

Furthermore, if the sequence $\left(n_{k}\right)$ is chosen to grow sufficiently rapidly, then

$$
P^{\Theta}(t)=P^{\Psi}(t)
$$

for all $t \geq 0$. Indeed, the systems $\Theta$ and $\Psi$ agree except at the times $n_{k}$, where the contribution to $Z_{n}^{\Psi}$ (with $n \geq n_{k}$ ) is

$$
Z_{1}^{\left(n_{k}\right)}(t)=\sum_{m \in I_{f}^{\left(n_{k}\right)}}\left\|D \varphi_{m}\right\|^{t}=\sum_{m=1}^{n_{k}}\left\|D \varphi_{m}\right\|^{t} \leq n_{k},
$$

while the contribution to $Z_{n}^{\Theta}$ is precisely $\left\|D \varphi_{\omega^{k}}\right\|^{t}$. Also observe that the sum $Z_{1}^{\left(n_{k}\right)}(t)$ in (11.3) is bounded below by $\left\|D \varphi_{1}\right\|^{d}$.

So let us suppose that $\left(n_{k}\right)$ is chosen such that $\log n_{k} \geq \max \left(k,\left|\log \left\|D \varphi_{\omega^{k}}\right\|\right|\right)$. Let $n \in \mathbb{N}$ be large, and let $k$ be maximal such that $n_{k} \leq n$. Then

$$
\begin{aligned}
\left|\log Z_{n}^{\Theta}(t)-\log Z_{n}^{\Psi}(t)\right| & \leq 2 k \log K+\sum_{j=1}^{k}\left|\log Z_{1}^{\left(n_{j}\right)}(t)\right|+\sum_{j=1}^{k} t\left|\log \left\|D \varphi_{\omega^{j}}\right\|\right| \\
& \leq 2 k \log K+k \cdot \log \left(n_{k}\right)+k \cdot d \cdot \log \left(n_{k}\right)=o(n),
\end{aligned}
$$

implying (11.2). By Theorem 1.1, it follows that

$$
\operatorname{HD}(J(\Phi)) \geq \operatorname{HD}(J(\Theta))=B(\Theta)=B(\Psi)=B(\Phi) \geq \operatorname{HD}(J(\Phi)),
$$

as claimed.

We can apply the same principle to subsets of Julia sets of certain holomorphic functions known as Ahlfors islands maps. For definitions, we refer to [13]. Here we note only that every non-constant, non-linear meromorphic function is an Ahlfors islands map; hence the following result implies Theorem 1.4.

11.2. Theorem (Subsets of Julia sets). Let $S$ be a compact Riemann surface, let $U \subset S$ be open and nonempty, and let $f: U \rightarrow S$ be a nonelementary Ahlfors islands map in the sense of Epstein [13, Definition 2.1].

Set

$$
J_{r, \text { dense }}(f):=\left\{z \in J_{r}(f): c l \bigcup_{j \geq 0} f^{j}(z)=J(f)\right\},
$$


where $J_{r}(f)$ is the radial Julia set of $f$ [13, Definition 2.5]. Then

$$
\operatorname{HD}\left(J_{r, \text { dense }}(f)\right)=\operatorname{HD}\left(J_{r}(f)\right)=\operatorname{HD}_{\text {hyp }}(f),
$$

where $\mathrm{HD}_{\text {hyp }}(f)$ denotes the hyperbolic dimension of $f$.

Proof. The second equality was proved in [13. It follows from the proof that, for every $h \in\left[0, \operatorname{HD}_{\text {hyp }}(f)\right)$, there is a finite conformal iterated function system $\Psi=\left\{\psi_{i}: i \in I\right\}$ with the following properties.

(a) $X$, the domain of the system $\Psi$, is a closed topological disk with analytic boundary.

(b) Each map $\psi_{i}, i \in I$, is a holomorphic inverse branch of some iterate of $f$, and extends to a conformal map defined on some larger disk $U \supset X$, independent of $i$.

(c) $\operatorname{HD}(J(\Psi)) \geq h$.

(d) For every $z \in J(f)$ and every $\varepsilon>0$ there exists an analytic inverse branch $\psi_{z, \varepsilon}: U \rightarrow S$ of some iterate of $f$ such that $\psi_{z, \varepsilon}(U) \subset B(z, \varepsilon)$. (To measure distances, we fix some metric on $S$; e.g. a conformal metric of constant curvature.)

Now choose a sequence $\left(z_{k}\right)_{k \in \mathbb{N}}$ in $J(f)$ that is dense in $J(f)$. Since $J(f)$ is uncountable, we may assume that no $z_{k}$ belongs to the orbit of a critical point. Now int $(X)$ intersects the Julia set of $f$ - indeed, $J(\Psi) \subset J(f)$ by definition - and hence the family $\left.f^{n}\right|_{\operatorname{int}(X)}$ omits at most finitely many values by the blowing-up property of the Julia set. Hence we can assume without loss of generality that each $z_{k}$ has an iterated preimage $\operatorname{in} \operatorname{int}(X)$. That is, for each $k$, there is some $m_{k}$ and an iterated preimage $\tilde{z}_{k} \in f^{-m_{k}}\left(z_{k}\right)$ such that $\tilde{z}_{k} \in \operatorname{int}(X)$. Let $\varepsilon=\varepsilon_{k} \leq 1 / k$ be so small that the branch $\vartheta_{k}$ of $f^{-m_{k}}$ that takes $z_{k}$ to $\tilde{z}_{k}$ is defined on $B\left(z_{k}, \varepsilon_{k}\right)$ and takes values in $\operatorname{int}(X)$ there.

Define

$$
\varphi_{k}:=\vartheta_{k} \circ \psi_{z_{k}, \varepsilon_{k}},
$$

let $\left(n_{k}\right)$ be a sufficiently growing sequence of positive integers, and set

$$
\Phi^{(n)}:= \begin{cases}\Psi & \text { if } n \neq n_{k} \text { for all } k \in \mathbb{N} \\ \left\{\varphi_{k}\right\} & \text { if } n=n_{k} \text { for some } k \in \mathbb{N} .\end{cases}
$$

By definition, the $f$-orbit of any point in $J(\Phi)$ passes through $B\left(z_{k}, 1 / k\right)$ for all $k$, and hence is dense in $J(\Phi)$. We also note that $J(\Phi) \subset J_{r}(f)$ by the definition of the radial Julia set.

The non-autonomous system $\Phi$ is uniformly finite, hence $\operatorname{HD}(J(\Phi))=B(\Phi)$ by Theorem 1.1 (or Corollary 4.3). If the sequence $\left(n_{k}\right)$ grows sufficiently quickly, then (as in the proof of the preceding theorem) $B(\Phi)=B(\Psi)=\operatorname{HD}(J(\Psi)) \geq h$. Since $h<\operatorname{HD}_{\text {hyp }}(f)$ was arbitrary, we are done.

\section{REFERENCES}

[1] R. Bowen, Hausdorff dimension of quasi-circles, Publ. Math. IHES,50 (1980), 11-25.

[2] Y. Dai, Z. Weng, L. Xi, and Y. Xiong, Quasisymmetrically minimal Moran sets and Hausdorff dimension, Annales Academiae Scientiarum Fennicae Mathematica, 36 (2011), 139151.

[3] M. Denker and Mariusz Urbański, On Sullivan's conformal measures for rational maps of the Riemann sphere, Nonlinearity 4 (1991), 365-384.

[4] D. Hensley, Continued fractions, World Scientific (2006). 
[5] M. Holland, Y. Zhang, Dimension results for inhomogeneous Moran set constructions, Preprint 2012.

[6] K. Falconer, Fractal Geometry, John Wiley \& Sons, 1990.

[7] T. Jordan and M. Rams, Increasing digit subsystems of infinite iterated function systems, Proc. Amer. Math. Soc. 140 (2012), 1267-1279.

[8] Tomasz Euczak, On the fractional dimension of sets of continued fractions, Mathematika 44 (1997), no. $1,5053$.

[9] D. Mauldin and Mariusz Urbański, Dimensions and measures in infinite iterated function systems, Proc. London Math. Soc. (3) 73 (1996) 105-154.

[10] D. Mauldin and Mariusz Urbański, Graph Directed Markov Systems: Geometry and Dynamics of Limit Sets, Cambridge University Press (2003)

[11] P.A.P. Moran, Additive functions of intervals and Hausdorff measure, Proc. Cambridge, Philos. Soc. 42 (1946), 15-23.

[12] F. Przytycki and Mariusz Urbański, Conformal Fractals Ergodic Theory Methods, Cambridge University Press (2010).

[13] L. Rempe, Hyperbolic dimension and radial Julia sets of transcendental functions, Proc. Amer. Math. Soc. 137 (2009), 1411-1420.

[14] M. Roy and Mariusz Urbański, Regularity properties of Hausdorff dimension in conformal infinite IFS, Ergodic Th. \& Dynam. Sys.25 (2005), 1961-1983.

[15] M. Roy, H. Sumi and Mariusz Urbański, Analytic Families of Holomorphic IFS, Nonlinearity 21 (2008), 2255-2279.

[16] M. Roy, H. Sumi and Mariusz Urbański, Lambda-topology vs. pointwise topology, Ergodic Th. and Dynam. Sys., 29 (2009), 685-713.

[17] Mario Roy and Mariusz Urbański, Random graph directed Markov systems, Discrete Contin. Dyn. Syst. 30 (2011), no. 1, 261-298.

[18] Mariusz Urbański and A. Zdunik, The finer geometry and dynamics of exponential family, Michigan Math. J. 51 (2003), 227-250.

[19] Zhiying Wen, Moran sets and Moran classes, Chinese Sci. Bull. 46 (2001), no. 22, 1849-1856.

Dept. of Mathematical Sciences, University of Liverpool, Liverpool L69 7ZL, UK

E-mail address: 1.rempe@liverpool.ac.uk

Department of Mathematics, University of North Texas, P.O. Box 311430, Denton, TX 76203-1430, USA

E-mail address: urbanski@unt.edu 